\title{
Synthesis and Metallation of Novel Fluorescent Conjugated Macrocycles
}

\author{
Cecily Ma, Andy Lo, Amir Abdolmaleki, and Mark J. MacLachlan* \\ Department of Chemistry, University of British Columbia, 2036 Main Mall, Vancouver, BC V6T 1Z1, \\ Canada \\ Email: mmaclach@chem.ubc.ca
}

\section{Supplementary Material}

\section{Experimental Section}

Materials Copper(I) iodide, Zinc(II) acetate dihydrate, phenylacetylene, and triphenylphosphine were obtained from Aldrich. Trans-dichlorobis(triphenylphosphine) palladium(II) was obtained from Strem Chemicals, Inc. Tetrahydrofuran (THF) was distilled from sodium / benzophenone ketal under nitrogen. Triethylamine was distilled from $\mathrm{NaOH}$ under nitrogen. Deuterated solvents were obtained from Cambridge Isotope Laboratories, Inc. Compounds 5a-c, 4-bromosalicylaldehyde and 1,4-diiodo-2,5dimethoxybenzene were prepared by literature methods. ${ }^{1-3}$

Equipment All reactions were carried out under nitrogen atmosphere unless otherwise noted. $300 \mathrm{MHz}$ ${ }^{1} \mathrm{H}$ NMR spectra and $75.5 \mathrm{MHz}{ }^{13} \mathrm{C}$ NMR spectra were recorded on a Bruker AV-300 spectrometer. ${ }^{1} \mathrm{H}$ and

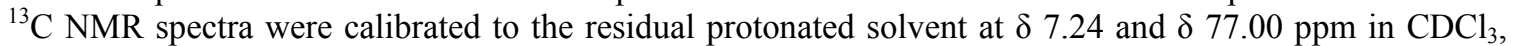
respectively. UV-vis spectra were obtained in THF (ca. $\times 10^{-6} \mathrm{M}$ ) on a Varian Cary 5000 UV-vis-near-IR spectrophotometer using a $1 \mathrm{~cm}$ quartz cuvette. IR spectra were obtained as $\mathrm{KBr}$ discs with a Bomems MBseries spectrometer. Fluorescence spectra were obtained in distilled THF on a Photon Technology International QuantaMaster fluorimeter using a $1 \mathrm{~cm}$ quartz cuvette. Quantum yield was referenced to a solution of anthracene in $\mathrm{EtOH}(\Phi=0.30) .{ }^{4}$ MALDI mass spectra were obtained in a dithranol matrix at the UBC Microanalytical Services Laboratory on a Micromass LCT time-of-flight (TOF) mass spectrometer. Electron Impact (EI) mass spectra and elemental analyses were also performed at the UBC Microanalytical Services laboratory. Melting points were obtained on a Fisher-John's melting point apparatus. Raman spectra were obtained on a Renishaw Raman microscope 1000 with excitation at $785 \mathrm{~nm}$. Molecular modeling and calculations were performed with Spartan '04.

4-Trimethylsilylacetylenesalicylaldehyde. Under a nitrogen atmosphere, 4-bromosalicylaldehyde (1.55 g, $7.7 \mathrm{mmol}), \mathrm{Pd}\left(\mathrm{PPh}_{3}\right)_{2} \mathrm{Cl}_{2}(0.11 \mathrm{~g}, 2 \mathrm{~mol} \%)$ and $\mathrm{PPh}_{3}(0.03 \mathrm{~g}, 1.5 \mathrm{~mol} \%)$ were dissolved in anhydrous THF (ca. $25 \mathrm{~mL})$, followed by the addition of $\operatorname{dry~} \mathrm{NEt}_{3}(2 \mathrm{~mL}, 15 \mathrm{mmol})$ and trimethylsilylacetylene $(1.2 \mathrm{~mL}$, $8.5 \mathrm{mmol})$, giving an orange solution. After stirring for $20 \mathrm{mins}$, CuI cocatalyst $(0.044 \mathrm{~g}, 3 \mathrm{~mol} \%)$ was added to the mixture, resulting in a dark brown solution. The reaction was stirred overnight at room temperature, and solvent was removed by rotary evaporation. The dark brown solid was dissolved in $n$ pentane and filtered through celite, giving a yellowish solution. Solvent was removed by rotary evaporation. Recrystallization from hexanes gave $1.48 \mathrm{~g}(88 \%)$ of yellow crystals. ${ }^{1} \mathrm{H} \mathrm{NMR}(300 \mathrm{MHz}$, $\left.\mathrm{CDCl}_{3}\right) \delta 10.97(\mathrm{~s}, 1 \mathrm{H}, \mathrm{OH}), 9.84(\mathrm{~s}, 1 \mathrm{H}, \mathrm{CH}=\mathrm{O}), 7.46\left(\mathrm{~d},{ }^{3} \mathrm{~J}_{\mathrm{HH}}=8.48 \mathrm{~Hz}, 1 \mathrm{H}\right.$, aromatic $\left.\mathrm{CH}\right), 7.06\left(\mathrm{~d},{ }^{3} \mathrm{~J}_{\mathrm{HH}}\right.$ $=8.01 \mathrm{~Hz}, 1 \mathrm{H}$, aromatic $\mathrm{CH}), 7.04(\mathrm{~s}, 1 \mathrm{H}$, aromatic $\mathrm{CH}), 0.24\left(\mathrm{~s}, 9 \mathrm{H}, \mathrm{Si}-\mathrm{CH}_{3}\right) \mathrm{ppm} ;{ }^{13} \mathrm{C} \mathrm{NMR}(75.5 \mathrm{MHz}$, $\left.\mathrm{CDCl}_{3}\right) \delta 195.74(\mathrm{CH}=\mathrm{O}), 133.31,123.28,120.78$, (aromatic $\left.C \mathrm{H}\right), 161.23,131.65,120.21$, (aromatic $C$ ), 103.52, $99.64(C \equiv C),-0.29\left(\mathrm{Si}_{-} \mathrm{CH}_{3}\right)$. EI-MS: $m / z=218\left(\mathrm{M}^{+}, 25 \%\right), 203\left(\mathrm{M}^{+}-\mathrm{CH}_{3}, 100 \%\right)$. UV-vis $\left(\mathrm{CH}_{2} \mathrm{Cl}_{2}\right): \lambda_{\max }(\varepsilon)=295\left(3.26 \times 10^{4}\right) \mathrm{nm}\left(\mathrm{L} \mathrm{mol}^{-1} \mathrm{~cm}^{-1}\right)$. IR $(\mathrm{KBr}): v=3216,2956,2159,1654,1620,1554$, 1493, 1296, 1248, 973, 858, $844 \mathrm{~cm}^{-1}$. Mp. 79-81 ${ }^{\circ}$ C. Anal. Calc'd for $\mathrm{C}_{12} \mathrm{H}_{14} \mathrm{O}_{2} \mathrm{Si}$ : C, 66.02; H, 6.46. Found: C, 65.92; H, 6.51.

4-Ethynylsalicylaldehyde. 4-Trimethylsilylacetylenesalicylaldehyde (1.98 g, $9.05 \mathrm{mmol})$ was dissolved in distilled THF (ca. $20 \mathrm{~mL}$ ). KOH (0.51 g, $9.05 \mathrm{mmol}$ ) was dissolved in $\mathrm{MeOH}$ (ca. $10 \mathrm{~mL})$ and added to the solution. The reaction mixture was stirred under air overnight at room temperature. Solvent was removed by rotary evaporation and extracted with $3 \times 200 \mathrm{~mL} \mathrm{CHCl}_{3}$. Acetic acid (ca. $0.5 \mathrm{~mL}$ ) was added to the 
aqueous layer and was further extracted with $3 \times 200 \mathrm{~mL}$ of $\mathrm{CHCl}_{3}$. The organic phases were combined and dried over $\mathrm{MgSO}_{4}$. The solution was filtered, followed by removal of solvent to obtain brown crystals. Recrystallization from hexanes afforded a yellow powder $(0.694 \mathrm{~g}, 53 \%) .{ }^{1} \mathrm{H} \mathrm{NMR}\left(300 \mathrm{MHz}, \mathrm{CDCl}_{3}\right) \delta$ $10.99(\mathrm{~s}, 1 \mathrm{H}, \mathrm{OH}), 9.87(\mathrm{~s}, 1 \mathrm{H}, \mathrm{CH}=\mathrm{O}), 7.50\left(\mathrm{~d},{ }^{3} \mathrm{~J}_{\mathrm{HH}}=8.35 \mathrm{~Hz}, 1 \mathrm{H}\right.$, aromatic $\left.\mathrm{CH}\right), 7.11-7.08(\mathrm{~m}, 2 \mathrm{H}$, aromatic $\mathrm{CH}), 3.27(\mathrm{~s}, 1 \mathrm{H},-\mathrm{C} \equiv \mathrm{C}-H) ;{ }^{13} \mathrm{C}$ NMR $\left(75.5 \mathrm{MHz}, \mathrm{CDCl}_{3}\right) 195.84(\mathrm{CH}=\mathrm{O}), 161.19,130.59$, 120.48 (aromatic $C$ ), 133.41, 123.41, 121.14 (aromatic $C H$ ), 82.34, $81.42(C \equiv C)$. EI-MS: $m / z=146\left(\mathrm{M}^{+}\right.$, $100 \%), 145\left(\mathrm{M}^{+}-\mathrm{H}, 78 \%\right)$. UV-vis $\left(\mathrm{CH}_{2} \mathrm{Cl}_{2}\right): \lambda_{\max }(\varepsilon)=283\left(2.24 \times 10^{4}\right) \mathrm{nm}\left(\mathrm{L} \mathrm{mol}^{-1} \mathrm{~cm}^{-1}\right)$. IR $(\mathrm{KBr}): v=$ $3277,2962,1659,1625,1556,1494,1262,1197,1125,1120,966,860,845,809,759 \mathrm{~cm}^{-1}$. Mp. $54-57^{\circ} \mathrm{C}$. Anal. Calc'd for $\mathrm{C}_{9} \mathrm{H}_{6} \mathrm{O}_{2}:$ C, 73.97; H, 4.14. Found: C, 73.57; H, 3.93.

Compound 2. Under a nitrogen atmosphere, 4-bromosalicylaldehyde (1.7 g, $8.4 \mathrm{mmol})$, 4ethynylsalicylaldehyde $(1.2 \mathrm{~g}, 8.4 \mathrm{mmol})$, triphenylphosphine $(0.03 \mathrm{~g}, 0.13 \mathrm{mmol})$ and transdichlorobis(triphenylphosphine) palladium(II) $(0.12 \mathrm{~g}, 0.17 \mathrm{mmol})$ were dissolved in $25 \mathrm{~mL}$ of anhydrous THF. Dry triethylamine $(4.7 \mathrm{~mL}, 34 \mathrm{mmol})$ was added, turning the solution from yellow to orange. After stirring for $20 \mathrm{mins}$, copper(I) iodide $(0.05 \mathrm{~g}, 0.25 \mathrm{mmol})$ was added, turning the solution to dark brown. After heating at reflux $\left(70^{\circ} \mathrm{C}\right)$ for 47 hours, the solution was cooled to room temperature. The yellow precipitate was isolated on a Buchner funnel and washed with cold dichloromethane. Yield: $0.865 \mathrm{~g}(39 \%)$. ${ }^{1} \mathrm{H}$ NMR $\left(300 \mathrm{MHz}, \mathrm{CDCl}_{3}\right) \delta 11.03(\mathrm{~s}, 2 \mathrm{H}, \mathrm{OH}), 9.89(\mathrm{~s}, 2 \mathrm{H}, \mathrm{CH}=\mathrm{O}), 7.54\left(\mathrm{~d},{ }^{3} \mathrm{~J}_{\mathrm{HH}}=8.37 \mathrm{~Hz}, 2 \mathrm{H}\right.$, aromatic $C \mathrm{H}), 7.17\left(\mathrm{dd},{ }^{3} \mathrm{~J}_{\mathrm{HH}}=8.10 \mathrm{~Hz},{ }^{4} \mathrm{~J}_{\mathrm{HH}}=1.26 \mathrm{~Hz}, 2 \mathrm{H}\right.$, aromatic $\left.\mathrm{CH}\right), 7.14(\mathrm{~s}, 2 \mathrm{H}$, aromatic $\mathrm{CH}) .{ }^{13} \mathrm{C}$ NMR (75.5 MHz, DMSO-d $\left.{ }_{6}\right) \delta 190.38(C \mathrm{H}=\mathrm{O}), 160.34,128.70,122.76$ (aromatic C), 129.13, 122.76, 119.81 (aromatic $C H), 91.34(C \equiv C)$. EI-MS: $m / z=266\left(\mathrm{M}^{+}, 100 \%\right), 265\left(\mathrm{M}^{+}-\mathrm{H}, 85 \%\right)$. UV-vis $\left(\mathrm{CH}_{2} \mathrm{Cl}_{2}\right)$ : $\lambda_{\max }(\varepsilon)=325\left(3.26 \times 10^{4}\right) \mathrm{nm}\left(\mathrm{L} \mathrm{mol}^{-1} \mathrm{~cm}^{-1}\right)$. IR $(\mathrm{KBr}): v=3206,2962,1659,1622,1631,1554,1498$, $1224,1184,1125,986,867,816,808 \mathrm{~cm}^{-1}$. Mp. $218-222{ }^{\circ} \mathrm{C}$. Anal. Calc'd for $\mathrm{C}_{16} \mathrm{H}_{10} \mathrm{O}$ : $: \mathrm{C}, 72.18 ; \mathrm{H}, 3.79$. Found: C, 71.77; H, 3.14

Compound 3. Under a nitrogen atmosphere, 4-ethynylsalicylaldehyde $(1.476 \mathrm{~g}, 12.1 \mathrm{mmol})$, transdichlorobis(triphenylphosphine) palladium(II) $(0.017 \mathrm{~g}, 0.024 \mathrm{mmol})$, copper(I) iodide $(0.023 \mathrm{~g}, 0.1 \mathrm{mmol})$ and benzoquinone $(1.177 \mathrm{~g}, 10.9 \mathrm{mmol})$ were dissolved in dry toluene $(\mathrm{ca} .60 \mathrm{~mL})$. Diisopropylamine $(0.018 \mathrm{mmol}, 2.5 \mathrm{~mL})$ was added to the mixture, which was then stirred overnight at room temperature. The reaction mixture was diluted with $150 \mathrm{~mL}$ water, and the aqueous layer was extracted with $3 \times 150 \mathrm{~mL}$ methylene chloride. The organic phases were combined and solvent was removed by rotary evaporation. The product was purified by filtration through a small plug of $\mathrm{SiO}_{2}$ using dichloromethane. Recrystallization from dichloromethane yielded the product as a brown solid $(0.521 \mathrm{~g}, 35 \%)$. ${ }^{1} \mathrm{H}$ NMR $(300$ $\left.\mathrm{MHz}, \mathrm{CDCl}_{3}\right) \delta 11.02(\mathrm{~s}, 2 \mathrm{H}, \mathrm{OH}), 9.89(\mathrm{~s}, 2 \mathrm{H}, \mathrm{CH}=\mathrm{O}), 7.53\left(\mathrm{~d},{ }^{3} \mathrm{~J}_{\mathrm{HH}}=8.43 \mathrm{~Hz}, 2 \mathrm{H}\right.$, aromatic $\left.\mathrm{CH}\right), 7.15$ $\left(\mathrm{dd},{ }^{3} \mathrm{~J}_{\mathrm{HH}}=8.043 \mathrm{~Hz},{ }^{4} \mathrm{~J}_{\mathrm{HH}}=1.38 \mathrm{~Hz}, 2 \mathrm{H}\right.$, aromatic $\left.\mathrm{CH}\right), 7.13(\mathrm{~s}, 2 \mathrm{H}$, aromatic $\mathrm{CH}) .{ }^{13} \mathrm{C}$ NMR $(75.5 \mathrm{MHz}$, $\left.\mathrm{CDCl}_{3}\right) \delta 195.78(\mathrm{CH}=\mathrm{O}), 161.18,129.67,120.87$ (aromatic $C$ ), 133.54, 123.74, 121.50 (aromatic $\mathrm{CH}$ ), 88.30, $77.23(C \equiv C)$. EI-MS: $\mathrm{m} / \mathrm{z}=290\left(\mathrm{M}^{+}, 100 \%\right)$. UV-vis $\left(\mathrm{CH}_{2} \mathrm{Cl}_{2}\right): \lambda_{\max }(\varepsilon)=338 \mathrm{~nm}\left(35000 \mathrm{~L} \mathrm{~mol}^{-}\right.$ $\left.{ }^{1} \mathrm{~cm}^{-1}\right)$. IR (KBr): $v=3179,2873,2348,1912,1660,1615,1554,1436,1270,1190,1123,967,868,795$, $761 \mathrm{~cm}^{-1}$. High res MS Calc'd for $\mathrm{C}_{18} \mathrm{H}_{10} \mathrm{O}_{4}: 290.05791$. Found: 290.0812

Compound 4. 4-Ethynylsalicylaldehyde $(0.500 \mathrm{~g}, 3.42 \mathrm{mmol})$, 1,4-diiodo-2,5-dimethoxybenzene $(0.651 \mathrm{~g}$, $1.67 \mathrm{mmol})$, trans-dichlorobis(triphenylphosphine) palladium(II) $(0.048 \mathrm{~g}, 2 \mathrm{~mol} \%)$, and triphenylphosphine $(0.013 \mathrm{~g}, 1.5 \mathrm{~mol} \%)$ were dissolved in dry THF (ca. $25 \mathrm{~mL}$ ) under nitrogen, followed by the addition of diisopropylamine (ca. $5 \mathrm{~mL}$ ). The mixture was stirred for 20 mins before the addition of copper(I) iodide $(0.019 \mathrm{~g}, 3 \mathrm{~mol} \%)$. The reaction mixture was heated to reflux overnight under nitrogen. After cooling to room temperature, the solvent was removed by rotary evaporation. The dark brown solid was dissolved in chloroform and was filtered through a short plug of silica gel to remove inorganic catalysts and salt. Recrystallization from methylene chloride yielded a yellow solid $(0.345 \mathrm{~g}, 24 \%) .{ }^{1} \mathrm{H}$ NMR $(300 \mathrm{MHz}$, $\left.\mathrm{CDCl}_{3}\right) \delta 11.04(\mathrm{~s}, 2 \mathrm{H}, \mathrm{OH}), 9.88(\mathrm{~s}, 2 \mathrm{H}, \mathrm{CH}=\mathrm{O}), 7.52\left(\mathrm{~d},{ }^{3} \mathrm{~J}_{\mathrm{HH}}=8.31 \mathrm{~Hz}, 2 \mathrm{H}\right.$, aromatic $\left.\mathrm{CH}\right), 7.19\left(\mathrm{dd},{ }^{3} \mathrm{~J}_{\mathrm{HH}}\right.$ $=8.39 \mathrm{~Hz},{ }^{4} \mathrm{~J}_{\mathrm{HH}}=1.51 \mathrm{~Hz}, 2 \mathrm{H}$, aromatic $\left.\mathrm{CH}\right), 7.16(\mathrm{~s}, 2 \mathrm{H}$, aromatic $\mathrm{CH}), 7.03(\mathrm{~s}, 2 \mathrm{H}$, aromatic $\mathrm{CH}), 3.90$ $\left(\mathrm{s}, 6 \mathrm{H}, \mathrm{OCH}_{3}\right){ }^{13} \mathrm{C}$ NMR $\left(75.5 \mathrm{MHz}, \mathrm{DMSO}-\mathrm{d}_{6}\right) \delta 190.46(\mathrm{CH}=\mathrm{O}), 160.56,153.89,129.71,122.63,112.68$ (aromatic $C$ ), 129.28, 122.58, 119.59, 115.95 (aromatic $\mathrm{CH}$ ), 94.26, $89.43(\mathrm{C} \equiv \mathrm{C}), 56.57\left(\mathrm{OCH}_{3}\right)$. EI-MS: $\mathrm{m} / \mathrm{z}=426\left(\mathrm{M}^{+}, 100 \%\right), 411\left(\mathrm{M}^{+}-\mathrm{CH}_{3}, 16 \%\right)$. UV-vis $\left(\mathrm{CH}_{2} \mathrm{Cl}_{2}\right): \lambda_{\max }(\varepsilon)=398\left(3.51 \times 10^{4}\right), 328(2.58 \mathrm{x}$ $\left.10^{4}\right), 295\left(1.86 \times 10^{4}\right) \mathrm{nm}\left(\mathrm{L} \mathrm{mol}^{-1} \mathrm{~cm}^{-1}\right)$. IR $(\mathrm{KBr}): v=3442,2925,2853,2215,1669,1621,1401,1236$, $1217,1041,974,818,793 \mathrm{~cm}^{-1}$. High res MS Calc'd for $\mathrm{C}_{26} \mathrm{H}_{18} \mathrm{O}_{6}$ : 426.11034 . Found: 426.11048 
Macrocycle 6. Under a nitrogen atmosphere, compound $2(0.106 \mathrm{~g}, 0.40 \mathrm{mmol})$ and 1,2-dioctyloxy-4,5diaminobenezne 5a $(0.145 \mathrm{~g}, 0.40 \mathrm{mmol})$ were combined in $15 \mathrm{~mL}$ of degassed $\mathrm{CHCl}_{3}$ and $5 \mathrm{~mL}$ of degassed $\mathrm{MeCN}$, resulting in a cloudy orange solution. The solution was heated to reflux $\left(90^{\circ} \mathrm{C}\right)$ for $24 \mathrm{~h}$, giving a clear, red solution. Upon cooling, a red powder precipitated, which was isolated on a Buchner funnel and washed with MeCN. Yield: $0.16 \mathrm{~g}(67 \%) .{ }^{1} \mathrm{H}$ NMR (300 MHz, THF-d $\left.\mathrm{d}_{8}\right) \delta 13.20(\mathrm{~s}, 6 \mathrm{H}, \mathrm{OH})$, $8.79(\mathrm{~s}, 6 \mathrm{H}, \mathrm{CH}=\mathrm{N}), 7.48\left(\mathrm{~d},{ }^{3} \mathrm{~J}_{\mathrm{HH}}=8.13 \mathrm{~Hz}, 6 \mathrm{H}\right.$, aromatic $\left.\mathrm{CH}\right), 7.15(\mathrm{~s}, 6 \mathrm{H}$, aromatic $\mathrm{CH}), 7.07-7.06(\mathrm{~m}$, $12 \mathrm{H}$, aromatic $\mathrm{CH}), 4.09\left(\mathrm{t},{ }^{3} \mathrm{~J}_{\mathrm{HH}}=6.33 \mathrm{~Hz}, 12 \mathrm{H}, \mathrm{OCH}_{2}\right), 1.80-0.89\left(\mathrm{~m}, 102 \mathrm{H}, \mathrm{OCH}_{2} \mathrm{C}_{7} H_{15}\right)$. MALDITOF-MS: $m / z=1785\left([\mathrm{M}+\mathrm{H}]^{+}\right)$. UV-vis $(\mathrm{THF}): \lambda_{\max }(\varepsilon)=406\left(8.37 \times 10^{4}\right), 373\left(7.89 \times 10^{4}\right), 333(6.15 \mathrm{x}$ $\left.10^{4}\right), 301\left(4.17 \times 10^{4}\right) \mathrm{nm}\left(\mathrm{L} \mathrm{mol}^{-1} \mathrm{~cm}^{-1}\right)$. IR $(\mathrm{KBr}): v=3457,2951,2924,2853,2131,1607,1522,1507$, 1493, 1264, 1199, 1116, 986, 871, $808 \mathrm{~cm}^{-1}$. Raman: $v=2208,1610,1586,1546,1521,1496,1437,1316$, 1237, 1187, $1095 \mathrm{~cm}^{-1}$. Mp. $>300{ }^{\circ} \mathrm{C}$. Anal. Calc'd for $\mathrm{C}_{114} \mathrm{H}_{138} \mathrm{~N}_{6} \mathrm{O}_{12} \cdot \mathrm{H}_{2} \mathrm{O}: \mathrm{C}, 75.97 ; \mathrm{H}, 7.83 ; \mathrm{N}, 4.66$. Found: C, 75.85; H, 7.93; N, 4.96. Fluorescence: $\Phi=0.14 \%$

Macrocycle 7. Compound 3 (0.150 g, $0.47 \mathrm{mmol})$ and 1,2-didecyloxy-4,5-diaminobenzene 5b (0.198 g, $0.47 \mathrm{mmol}$ ) were combined in a $100 \mathrm{~mL}$ Schlenk flask under nitrogen. Degassed acetonitrile (ca. $4 \mathrm{~mL}$ ) and degassed chloroform (ca. $12 \mathrm{~mL}$ ) were added to the flask, giving a cloudy orange solution. The solution was heated to reflux overnight. Upon cooling to room temperature, a red solid precipitated, which was isolated on a Buchner funnel and washed with MeCN. Yield: $0.216 \mathrm{~g}(62 \%)$. ${ }^{1} \mathrm{H}$ NMR (300 MHz, THF$\left.\mathrm{d}_{8}\right) \delta 13.19(\mathrm{~s}, 6 \mathrm{H}, \mathrm{OH}), 8.79(\mathrm{~s}, 6 \mathrm{H}, \mathrm{CH}=\mathrm{N}), 7.48\left(\mathrm{~d},{ }^{3} \mathrm{~J}_{\mathrm{HH}}=8.28 \mathrm{~Hz}, 6 \mathrm{H}\right.$, aromatic $\left.\mathrm{CH}\right), 7.14(\mathrm{~s}, 6 \mathrm{H}$, aromatic $\mathrm{CH}), 7.09-7.06(\mathrm{~m}, 12 \mathrm{H}$, aromatic $\mathrm{CH}), 4.08\left(\mathrm{t},{ }^{3} \mathrm{~J}_{\mathrm{HH}}=6.42 \mathrm{~Hz}, 12 \mathrm{H}, \mathrm{OCH}_{2}\right), 1.94-0.86(\mathrm{~m}$, $\left.126 \mathrm{H}, \mathrm{OCH}_{2} \mathrm{C}_{9} H_{19}\right)$. MALDI-TOF-MS: $m / z=2025\left([\mathrm{M}+\mathrm{H}]^{+}, 100\right), 2047\left([\mathrm{M}+\mathrm{Na}]^{+}, 12\right), 2082$ $\left(\left[\mathrm{M}+\mathrm{H}_{2} \mathrm{O}+\mathrm{K}\right]^{+}, 15\right) . \mathrm{UV}$-vis $(\mathrm{DCM}): \lambda_{\max }(\varepsilon)=374\left(1.32 \times 10^{4}\right) . \mathrm{IR}(\mathrm{KBr}): v=3457,2951,2924,2853$, 2131, 1607, 1522, 1507, 1493, 1264, 1199, 1116, 986, 871, $808 \mathrm{~cm}^{-1}$. Raman: $v=2212,1615,1605,1585$, $1574,1540,1518,1501,1434,1381,1343,1308,1267,1239,1184,1132,1019,905,817,592,577,512$, 484, $413 \mathrm{~cm}^{-1}$. Anal. Calc'd for $\mathrm{C}_{132} \mathrm{H}_{162} \mathrm{~N}_{6} \mathrm{O}_{12} \cdot \mathrm{H}_{2} \mathrm{O}$ : C, 77.61; H, 8.09; N, 4.11. Found: C, 77.31; H, 7.89; $\mathrm{N}, 4.36$. Fluorescence: $\Phi=0.15 \%$

Macrocycle 8. Under a nitrogen atmosphere, compound $4(0.232 \mathrm{~g}, 0.54 \mathrm{mmol})$ and 1,2-dioctyloxy-4,5diaminobenezne 5a $(0.198 \mathrm{~g}, 0.54 \mathrm{mmol})$ were dissolved in $18 \mathrm{~mL}$ of degassed $\mathrm{CHCl}_{3}$ and $6 \mathrm{~mL}$ of degassed $\mathrm{MeCN}$. The solution was heated to reflux $\left(90^{\circ} \mathrm{C}\right)$ for $24 \mathrm{~h}$, then cooled to room temperature, yielding red powder. The red solid, macrocycle 8, was isolated on a Buchner funnel, washed with cold $\mathrm{MeCN}$, and dried under vacuum. Yield: $0.17 \mathrm{~g}(40 \%) .{ }^{1} \mathrm{H}$ NMR $\left(300 \mathrm{MHz}, \mathrm{THF}-\mathrm{d}_{8}\right) \delta 13.16(\mathrm{~s}, 6 \mathrm{H}, \mathrm{OH})$, $8.78(\mathrm{~s}, 6 \mathrm{H}, \mathrm{CH}=\mathrm{N}), 7.47\left(\mathrm{~d},{ }^{3} \mathrm{~J}_{\mathrm{HH}}=8.31 \mathrm{~Hz}, 6 \mathrm{H}\right.$, aromatic $\left.\mathrm{CH}\right), 7.13-7.04(\mathrm{~m}, 24 \mathrm{H}$, aromatic $\mathrm{CH}), 4.09(\mathrm{t}$, $\left.{ }^{3} \mathrm{~J}_{\mathrm{HH}}=6.26 \mathrm{~Hz}, 12 \mathrm{H}, \mathrm{OCH}_{2}\right), 3.90\left(\mathrm{~s}, 18 \mathrm{H},-\mathrm{OCH}_{3}\right), 1.7-0.8\left(\mathrm{~m}, 102 \mathrm{H}, \mathrm{OCH}_{2} \mathrm{C}_{7} H_{15}\right)$. MALDI-TOF-MS: $\mathrm{m} / \mathrm{z}=2265\left([\mathrm{M}+\mathrm{H}]^{+}, 100\right), 2326(10) . \mathrm{UV}$-vis (THF): $\lambda_{\max }(\varepsilon)=408\left(1.87 \times 10^{4}\right), 329\left(9.02 \times 10^{4}\right) \mathrm{nm}(\mathrm{L}$ $\mathrm{mol}^{-1} \mathrm{~cm}^{-1}$ ). IR (KBr): $v=3428,2923,2851,2202,1608,1490,1263,1217,1039,804 \mathrm{~cm}^{-1}$. Raman: $v=$ 2201, 1603, 1587, 1552, 1513, 1432, 1363, 1315, 1272, 1237, 1178, $1079 \mathrm{~cm}^{-1}$. Anal. Calc'd for $\mathrm{C}_{144} \mathrm{H}_{162} \mathrm{~N}_{6} \mathrm{O}_{18} \cdot 5 \mathrm{H}_{2} \mathrm{O}: \mathrm{C}, 73.44 ; \mathrm{H}, 7.36 ; \mathrm{N}, 3.57$. Found: $\mathrm{C}, 73.37 ; \mathrm{H}, 7.16 ; \mathrm{N}, 3.94$. Fluorescence: $\Phi=$ $0.13 \%$.

Macrocycle 9. Under a nitrogen atmosphere, macrocycle $6(0.102 \mathrm{~g}, 0.05 \mathrm{mmol})$ and $\mathrm{Zn}(\mathrm{OAc})_{2} \cdot 2 \mathrm{H}_{2} \mathrm{O}$ $(0.040 \mathrm{~g}, 0.18 \mathrm{mmol})$ were dissolved in $20 \mathrm{~mL}$ of dry THF. After heating at reflux $\left(70^{\circ} \mathrm{C}\right)$ for $2 \mathrm{~h}$, the red solution was cooled to room temperature. The solvent was evacuated and macrocycle 9 was isolated on a Buchner funnel and washed with hot MeOH. Yield: 0.090g (80 \%). ${ }^{1} \mathrm{H}$ NMR (300 MHz, THF-d 8 ) $\delta 8.85$ (s, $6 \mathrm{H}, \mathrm{CH}=\mathrm{N}), 7.40(\mathrm{~s}, 6 \mathrm{H}$, aromatic $\mathrm{CH}), 7.24\left(\mathrm{~d},{ }^{3} \mathrm{~J}_{\mathrm{HH}}=8.39 \mathrm{~Hz}, 6 \mathrm{H}\right.$, aromatic $\left.\mathrm{CH}\right), 7.16(\mathrm{~s}, 6 \mathrm{H}$, aromatic $\mathrm{CH}), 6.56\left(\mathrm{~d},{ }^{3} \mathrm{~J}_{\mathrm{HH}}=8.55 \mathrm{~Hz}, 6 \mathrm{H}\right.$, aromatic $\left.\mathrm{CH}\right), 4.13\left(\mathrm{t},{ }^{3} \mathrm{~J}_{\mathrm{HH}}=6.60 \mathrm{~Hz}, 12 \mathrm{H}, \mathrm{OCH}_{2}\right), 1.94-0.90(\mathrm{~m}, 102 \mathrm{H}$, $\left.\mathrm{OCH}_{2} \mathrm{C}_{7} H_{15}\right)$. MALDI-TOF-MS: $\mathrm{m} / \mathrm{z}=1974\left([\mathrm{M}+\mathrm{H}]^{+}\right), 1972\left([\mathrm{M}+\mathrm{H}-\mathrm{Zn}]^{+}\right), 1848\left([\mathrm{M}+\mathrm{H}-2 \mathrm{Zn}]^{+}\right), 1785$ $\left([\mathrm{M}+\mathrm{H}-3 \mathrm{Zn}]^{+}\right) \mathrm{UV}$-vis $(\mathrm{THF}): \lambda_{\max }(\varepsilon)=481\left(9.08 \times 10^{4}\right), 442\left(1.33 \times 10^{5}\right), 379\left(8.64 \times 10^{4}\right), 353(9.73 \mathrm{x}$ $\left.10^{4}\right) \mathrm{nm}\left(\mathrm{L} \mathrm{mol}^{-1} \mathrm{~cm}^{-1}\right)$. IR (KBr): $v=3438,2950,2924,2854,2131,1606,1586,1507,1481,1467,1384$, 1375, 1268, 1170, $1134 \mathrm{~cm}^{-1}$. Mp. $>300{ }^{\circ} \mathrm{C}$. Anal. Calc'd for $\mathrm{C}_{114} \mathrm{H}_{132} \mathrm{~N}_{6} \mathrm{O}_{12} \mathrm{Zn}_{3} \cdot 9 \mathrm{H}_{2} \mathrm{O}: \mathrm{C}, 64.08 ; \mathrm{H}, 7.08$; N, 3.93. Found: C, 63.95; H, 7.08; N, 4.12. ${ }^{5}$ Fluorescence: $\Phi=0.86 \%$.

Macrocycle 10. Macrocycle $7(0.100 \mathrm{~g}, 0.049 \mathrm{mmol})$ and $\mathrm{Zn}(\mathrm{OAc})_{2} \cdot 2 \mathrm{H}_{2} \mathrm{O}(0.033 \mathrm{~g}, 0.158 \mathrm{mmol})$ were added to $30 \mathrm{~mL}$ dry THF in a $100 \mathrm{~mL}$ Schlenk flask. The solution was heated to reflux for 3 hours. After solvent was removed under reduced pressure, remaining solid was washed with hot methanol followed by filtration to yield dark red solid as product $(0.073 \mathrm{~g}, 55 \%) .{ }^{1} \mathrm{H}$ NMR $\left(300 \mathrm{MHz}, \mathrm{THF}-\mathrm{d}_{8}\right) \delta 8.85(\mathrm{~s}, 6 \mathrm{H}$, 
$\mathrm{C} H=\mathrm{N}), 7.40(\mathrm{~s}, 6 \mathrm{H}$, aromatic $\mathrm{CH}), 7.24\left(\mathrm{~d},{ }^{3} \mathrm{~J}_{\mathrm{HH}}=8.39 \mathrm{~Hz}, 6 \mathrm{H}\right.$, aromatic $\left.\mathrm{CH}\right), 7.16(\mathrm{~s}, 6 \mathrm{H}$, aromatic $\mathrm{CH})$, $6.56\left(\mathrm{~d},{ }^{3} \mathrm{~J}_{\mathrm{HH}}=8.55 \mathrm{~Hz}, 6 \mathrm{H}\right.$, aromatic $\left.\mathrm{CH}\right), 4.13\left(\mathrm{t},{ }^{3} \mathrm{~J}_{\mathrm{HH}}=6.60 \mathrm{~Hz}, 12 \mathrm{H}, \mathrm{OCH}_{2}\right), 1.94-0.90(\mathrm{~m}, 126 \mathrm{H}$, $\left.\mathrm{OCH}_{2} \mathrm{C}_{9} H_{19}\right)$. MALDI-TOF-MS: $\mathrm{m} / \mathrm{z}=2216\left([\mathrm{M}+\mathrm{H}]^{+}, 100\right), 2152\left([\mathrm{M}+\mathrm{H}-\mathrm{Zn}]^{+}, 30\right), 2089\left([\mathrm{M}+\mathrm{H}-2 \mathrm{Zn}]^{+}\right.$, 15), 2504 (see ref. 6, 25). UV-vis (THF): $\lambda_{\max }(\varepsilon)=446(12900) \mathrm{nm}\left(\mathrm{L} \mathrm{mol}^{-1} \mathrm{~cm}^{-1}\right)$. IR $(\mathrm{KBr}): v=3439$, 2924, 2853, 2854, 2347, 2207, 1607, 1586, 1502, 1466, 1384, 1207, 1129, 981, $871 \mathrm{~cm}^{-1} . \mathrm{C}_{132} \mathrm{H}_{156} \mathrm{~N}_{6} \mathrm{O}_{12}$ $\mathrm{Zn}_{3} \cdot 9 \mathrm{H}_{2} \mathrm{O}: \mathrm{C}, 66.70 ; \mathrm{H}, 7.38 ; \mathrm{N}, 3.54$. Found: C, 66.52; H, 7.12; N, 3.82. ${ }^{5}$ Fluorescence: $\Phi=0.92 \%$.

Macrocycle 11. Under a nitrogen atmosphere, macrocycle $8(0.165 \mathrm{~g}, 0.07 \mathrm{mmol})$ and $\mathrm{Zn}(\mathrm{OAc})_{2} \cdot 2 \mathrm{H}_{2} \mathrm{O}$ $(0.051 \mathrm{~g}, 0.23 \mathrm{mmol})$ were dissolved in dry THF (ca. $25 \mathrm{~mL})$. The solution was heated to reflux for 80 minutes. After solvent was removed under reduced pressure, compound $\mathbf{1 1}$ was isolated on a Buchner funnel and washed with hot methanol, giving dark red solid as product $(0.139 \mathrm{~g}, 78 \%)$. ${ }^{1} \mathrm{H}$ NMR (300 $\left.\mathrm{MHz}, \mathrm{THF}-\mathrm{d}_{8}\right) \delta 8.87(\mathrm{~s}, 6 \mathrm{H}, \mathrm{CH}=\mathrm{N}), 7.38(\mathrm{~s}, 6 \mathrm{H}$, aromatic $\mathrm{CH}), 7.25\left(\mathrm{~d},{ }^{3} \mathrm{~J}_{\mathrm{HH}}=8.16 \mathrm{~Hz}, 6 \mathrm{H}\right.$, aromatic $\mathrm{CH}), 7.12(\mathrm{~s}, 6 \mathrm{H}$, aromatic $\mathrm{CH}), 7.06(\mathrm{~s}, 6 \mathrm{H}$, aromatic $\mathrm{CH}), 6.59\left(\mathrm{~d},{ }^{3} \mathrm{~J}_{\mathrm{HH}}=8.39 \mathrm{~Hz}, 6 \mathrm{H}\right.$, aromatic $\left.\mathrm{CH}\right)$, $4.13\left(\mathrm{t},{ }^{3} \mathrm{~J}_{\mathrm{HH}}=5.58 \mathrm{~Hz}, 12 \mathrm{H}, \mathrm{OCH}_{2}\right), 3.93\left(\mathrm{~s}, 18 \mathrm{H}, \mathrm{OCH}_{3}\right), 1.94-0.90\left(\mathrm{~m}, 102 \mathrm{H}, \mathrm{OCH}_{2} \mathrm{C}_{7} H_{15}\right)$. MALDITOF-MS: $\mathrm{m} / \mathrm{z}=2456\left([\mathrm{M}+\mathrm{H}]^{+}, 100\right), 2392\left([\mathrm{M}+\mathrm{H}-\mathrm{Zn}]^{+}, 35\right), 2744$ (see ref. 6, 20), $1196\left([\mathrm{M}+2 \mathrm{H}]^{2+}, 20\right)$. UV-vis (THF): $\lambda_{\max }(\varepsilon)=438\left(1.83 \times 10^{5}\right), 337\left(8.54 \times 10^{5}\right) \mathrm{nm}\left(\mathrm{L} \mathrm{mol}^{-1} \mathrm{~cm}^{-1}\right)$. IR $(\mathrm{KBr}): v=3423,2923$, 2852, 2385, 2348, 1606, 1586, 1468, 1384, 1267, 1216, 1171, $796 \mathrm{~cm}^{-1}$. Anal. Calc'd for $\mathrm{C}_{144} \mathrm{H}_{156} \mathrm{~N}_{6} \mathrm{O}_{18}$ $\mathrm{Zn}_{3} \cdot 8 \mathrm{H}_{2} \mathrm{O}: \mathrm{C}, 66.54 ; \mathrm{H}, 6.67 ; \mathrm{N}, 3.23$. Found: C, 66.04; H, 6.03; N, 3.35. ${ }^{5}$ Fluorescence: $\Phi=0.90 \%$.

Compound 12. 4-Bromosalicylaldehyde $(0.100 \mathrm{~g}, 0.498 \mathrm{mmol})$, ethynylbenzene $(0.050 \mathrm{~g}, 0.498 \mathrm{mmol})$, trans-dichlorobis(triphenylphoshphine)palladium(II) $(0.035 \mathrm{~g}, 2 \mathrm{~mol} \%)$, and triphenylphosphine $(0.039 \mathrm{~g}, 3$ mol\%) were dissolved in dry THF (ca. $10 \mathrm{~mL}$ ) under nitrogen, followed by the addition of dry $\mathrm{NEt}_{3}$ (ca. 2 $\mathrm{mL})$. The mixture was stirred for 20 mins at RT before the addition of copper iodide $(0.047 \mathrm{~g}, 5 \mathrm{~mol} \%)$. The reaction mixture was then heated to reflux overnight under nitrogen. After cooling to room temperature, the solvent was removed by rotary evaporation. The dark brown solid was dissolved in toluene and filtered through a plug of silica gel to remove inorganic catalysts and salt. Recrystallization from petroleum ether yielded a yellow solid $(0.11 \mathrm{~g}, 92 \%) .{ }^{1} \mathrm{H}$ NMR $\left(300 \mathrm{MHz}, \mathrm{CDCl}_{3}\right) \delta 11.03(\mathrm{~s}, 1 \mathrm{H}, \mathrm{OH}), 9.87(\mathrm{~s}, 1 \mathrm{H}, \mathrm{CH}=\mathrm{O})$, $7.54\left(\mathrm{~d}, 1 \mathrm{H}\right.$, aromatic $\left.\mathrm{CH}, J_{\mathrm{H}-\mathrm{H}}=2.3 \mathrm{~Hz}\right), 7.52(\mathrm{t}, 2 \mathrm{H}$, aromatic $\mathrm{CH}), 7.50(\mathrm{~s}, 1 \mathrm{H}$, aromatic $\mathrm{CH}), 7.37(\mathrm{~d}, 2 \mathrm{H}$, aromatic $\left.\mathrm{CH}, J_{\mathrm{H}-\mathrm{H}}=2.5 \mathrm{~Hz}\right), 7.35(\mathrm{~s}, 1 \mathrm{H}$, aromatic $\mathrm{CH}), 7.14(\mathrm{~s}, 1 \mathrm{H}$, aromatic $\mathrm{CH}), 7.12(\mathrm{~s}, 1 \mathrm{H}$, aromatic CH). EI-MS: $\mathrm{m} / \mathrm{z}=222\left(\mathrm{M}^{+}, 100 \%\right)$. IR (KBr): $v=3064,2208,16.58,1643,1621,1549,1505,1341,1319$, 1244, 1226, 1198, 968, 886, 825, 812, 755. Anal.Calc'd for $\mathrm{C}_{15} \mathrm{H}_{10} \mathrm{O}_{2}$ : C, 81.07; H, 4.54. Found: C, 81.24; H, 4.62 .

Model Compound 13. In a $100 \mathrm{~mL}$ Schlenk flask, compound $12(0.20 \mathrm{~g}, 0.90 \mathrm{mmol}), 1,2-(2-$ ethylhexyloxy)-4,5-diaminobenzene $5 \mathbf{c}(0.16 \mathrm{~g}, 0.45 \mathrm{mmol})$ and $\mathrm{Zn}(\mathrm{OAc})_{2} 2 \mathrm{H}_{2} \mathrm{O}(0.142 \mathrm{~g}, 0.54 \mathrm{mmol})$ were combined under nitrogen. Dry THF (ca. $10 \mathrm{~mL}$ ) were added to the flask and the solution was heated to reflux overnight. The red solution was cooled to room temperature; upon adding $\mathrm{MeOH}$, orange solid precipitated, which was isolated on a Buchner funnel, washed with cold $\mathrm{MeOH}$, and dried under vacuum. Yield 0.357g (95\%). ${ }^{1} \mathrm{H}$ NMR $\left(300 \mathrm{MHz}\right.$, THF- $\left.\mathrm{d}_{8}\right) \delta 8.84(\mathrm{~s}, 2 \mathrm{H}, \mathrm{CH}=\mathrm{N}), 7.52\left(\mathrm{~d}, 2 \mathrm{H}\right.$, aromatic $\mathrm{CH}, J_{\mathrm{H}-}$ $\left.\mathrm{H}_{\mathrm{H}}=2.2 \mathrm{~Hz}\right), 7.49\left(\mathrm{~d}, 2 \mathrm{H}\right.$, aromatic $\left.\mathrm{CH}, J_{\mathrm{H}-\mathrm{H}}=1.6 \mathrm{~Hz}\right), 7.38(\mathrm{~s}, 2 \mathrm{H}$, aromatic $\mathrm{CH}), 7.35(\mathrm{~s}, 2 \mathrm{H}$, aromatic $\mathrm{CH})$, $7.33\left(\mathrm{~d}, 4 \mathrm{H}\right.$, aromatic $\left.\mathrm{CH}, J_{\mathrm{H}-\mathrm{H}}=1.3 \mathrm{~Hz}\right), 7.25\left(\mathrm{~d}, 2 \mathrm{H}\right.$, aromatic $\left.\mathrm{CH}, J_{\mathrm{H}-\mathrm{H}}=8.1 \mathrm{~Hz}\right), 6.97(\mathrm{~d}, 2 \mathrm{H}$, aromatic $\left.\mathrm{CH}, J_{\mathrm{H}-\mathrm{H}}=1.2 \mathrm{~Hz}\right), 6.60-6.57\left(\mathrm{dd}, 2 \mathrm{H}\right.$, aromatic $\left.\mathrm{CH}, J_{\mathrm{H}-\mathrm{H}}=8.0 \mathrm{~Hz}, J_{\mathrm{H}-\mathrm{H}}=1.5 \mathrm{~Hz}\right), 4.05-4.03\left(\mathrm{~d}, 4 \mathrm{H}, \mathrm{OCH}_{2}, J_{\mathrm{H}-}\right.$ $\mathrm{H}=5.2 \mathrm{~Hz}), 1.77-0.89\left(\mathrm{~m}, 30 \mathrm{H}, \mathrm{OCH}_{2}-\mathrm{C}_{7} \mathrm{H}_{15}\right)$. EI-MS: $\mathrm{m} / \mathrm{z}=834\left(\mathrm{M}^{+}, 60 \%\right)$. UV-vis (THF): $\lambda_{\max }(\varepsilon)=405$ $\left(3.75 \times 10^{4}\right) \mathrm{nm}\left(\mathrm{L} \mathrm{mol}^{-1} \mathrm{~cm}^{-1}\right)$. IR $(\mathrm{KBr}): v=3433,2957,2928,2870,2381,2348,2310,1605,1503,1467$, 1381, 1268, 1177, 1115, 980, 824, $791 \mathrm{~cm}^{-1}$. Anal.Calc'd for $\mathrm{C}_{52} \mathrm{H}_{54} \mathrm{~N}_{2} \mathrm{O}_{4} \mathrm{Zn} \cdot 2 \mathrm{H}_{2} \mathrm{O}: \mathrm{C}, 71.59 ; \mathrm{H}, 6.70 ; \mathrm{N}$, 3.21. Found: C, $72.21 ; \mathrm{H}, 6.59 ; \mathrm{N}, 3.62$. Fluorescence : $\Phi=0.94 \%$.

Metal Titration Experiments. Titration experiments were preformed by adding aliquots (via microliter syringe) of a metal salt $\left(\mathrm{Zn}(\mathrm{OAc})_{2} \cdot 2 \mathrm{H}_{2} \mathrm{O}\right.$ in THF or $\mathrm{Ni}(\mathrm{OAc})_{2}$ in $\left.\mathrm{EtOH} ; 8 \times 10^{-5} \mathrm{M}\right)$ to a quartz cuvette containing ca. $1 \times 10^{-6} \mathrm{M}$ of macrocycle in $\sim 3.5 \mathrm{~mL}$ of THF.

\section{References}

1. Kim, D.-H.; Choi, M. J.; Chang, S.-K. Bull. Korean Chem. Soc. 2000, 21, 145.

2. Tromelin, A.; Demerseman, P.; Royer, R.; Gayral, P.; Fourniat, J. Eur. J. Med. Chem. 1986, 21, 397. 
3. Waybright, S. M.; Singleton, C. P.; Wachter, K.; Murphy, C. J.; Bunz, U. H. F. J. Am. Chem. Soc. 2001, $123,1828$.

4. Parker, C. A. Photoluminescence of Solutions, $1^{\text {st }}$ ed.; Elsevier Publishing Company: Amsterdam, 1968; p 261.

5. Elemental analysis of the metallated macrocycles has been problematic. Although the ${ }^{1} \mathrm{H}$ NMR spectrum of the macrocycles indicates that they are pure, they consistently give $\mathrm{C}$ analyses that are $6-8 \%$ low, while the $\mathrm{H}$ and $\mathrm{N}$ analyses are quite close. We have rationalized this as being due to additional water molecules that are coordinated to the metals and/or occupy the space in the macrocycoes; we do see these in the IR and NMR spectra ( $2.5 \mathrm{ppm}$ in THF- $\left.\mathrm{d}_{8}\right)$. However, even with monometallic salphen complexes we consistently obtain analyses that are several percent low in C. We are working on this problem, but are confident that it is an issue related to the combustion of these compounds. See Dai, Y.; Katz, T.J. J. Org. Chem. 1997, 62, 1274 (and references therein) for similar cases with conjugated oligomers and polymers, some containing salen-type ligands.

6. In the MALDI-TOF spectra of some $\mathrm{Zn}$ complexes, we observe a peak at $m / z=288$ above the $[\mathrm{M}+\mathrm{H}]^{+}$ peak. This appears to be a complex formed with the dithranol matrix (e.g., $[\mathrm{M}-\mathrm{H}+\text { dithranol }+\mathrm{Zn}]^{+}$). Dithranol complexes of macrocycles have been observed before: Höger et al. Macromolecules 1997, 30, 3110-3111.

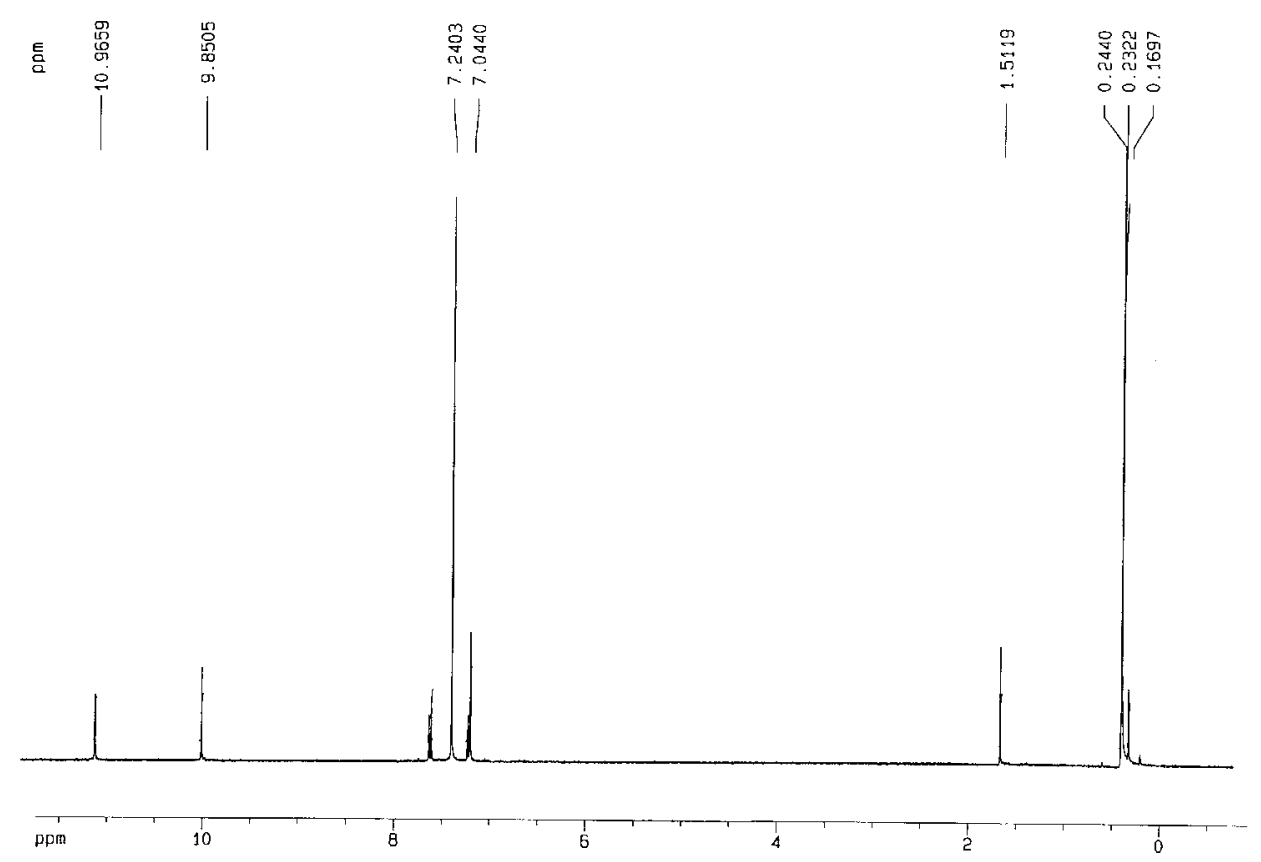

Figure S1. ${ }^{1}$ H NMR spectrum of 4-trimethylsilylacetylenesalicylaldehyde. 


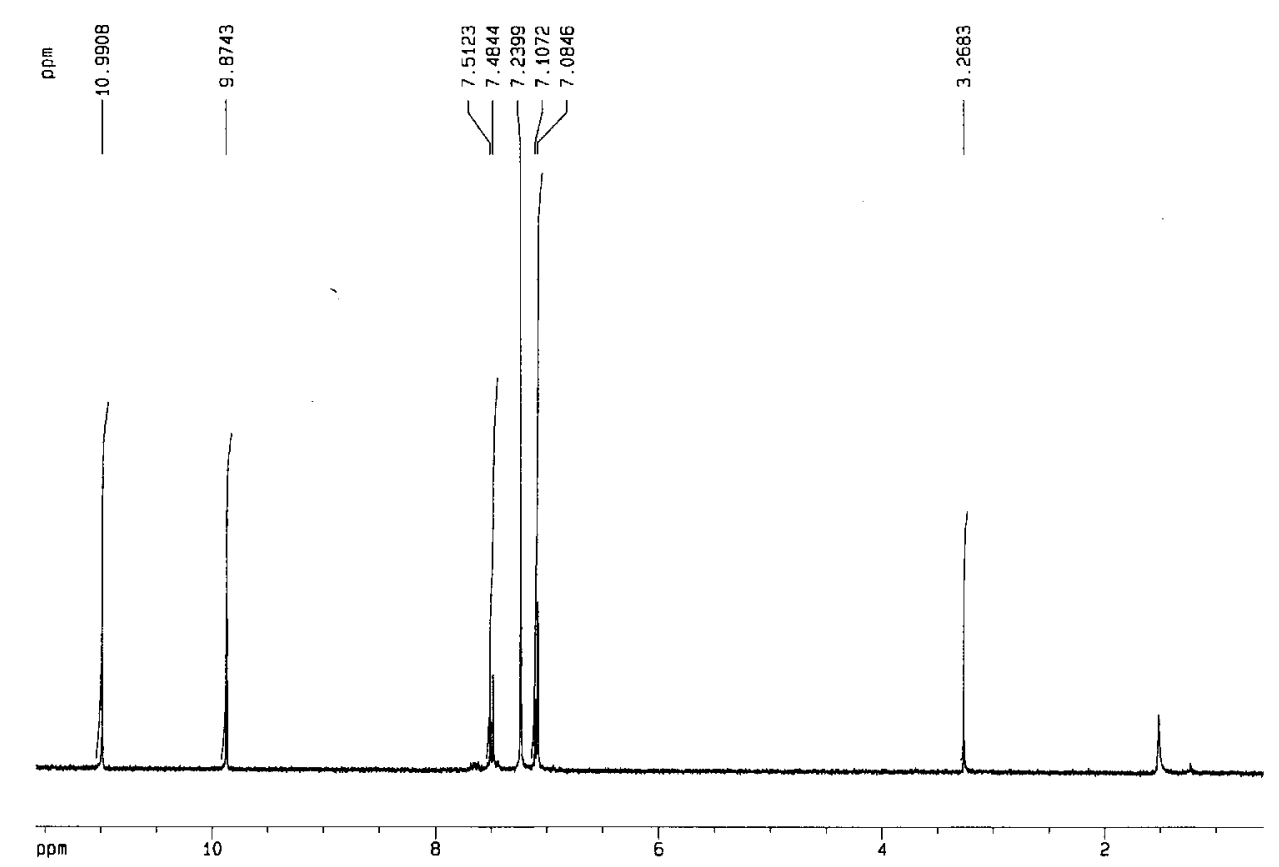

Figure S2. ${ }^{1}$ H NMR spectrum of 4-ethynylsalicylaldehyde.

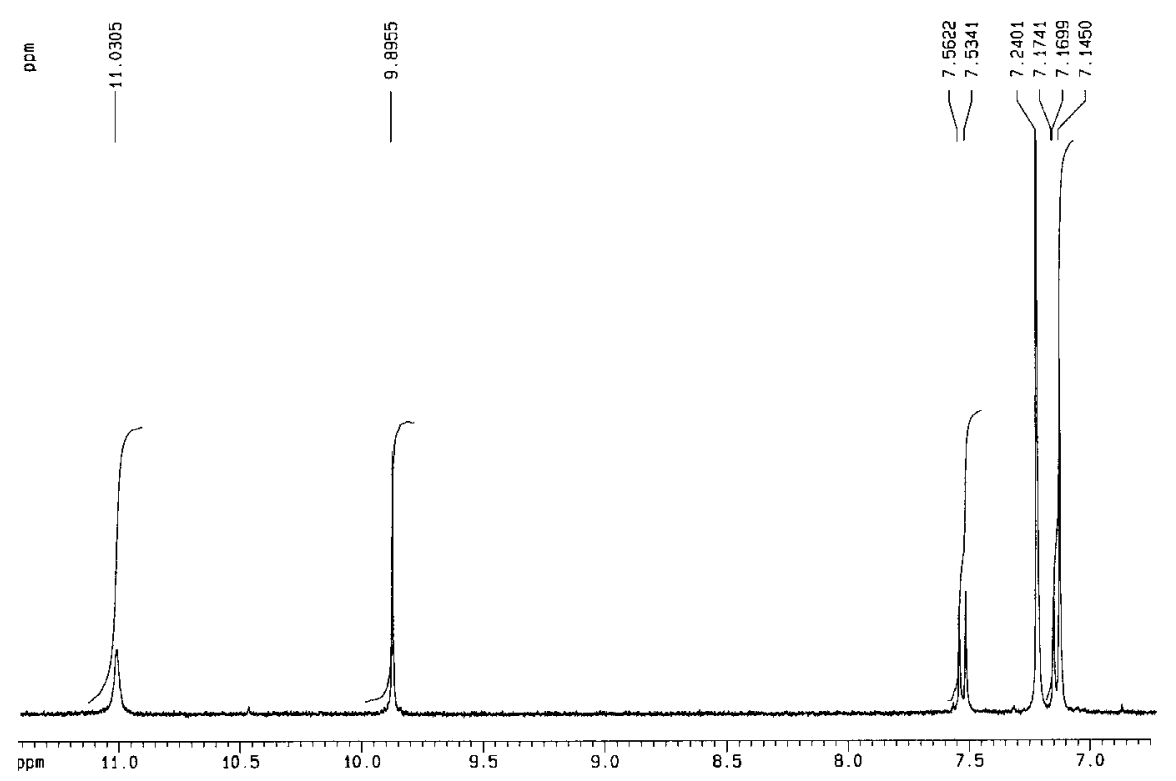

Figure S3. ${ }^{1} \mathrm{H}$ NMR spectrum of compound 2. 


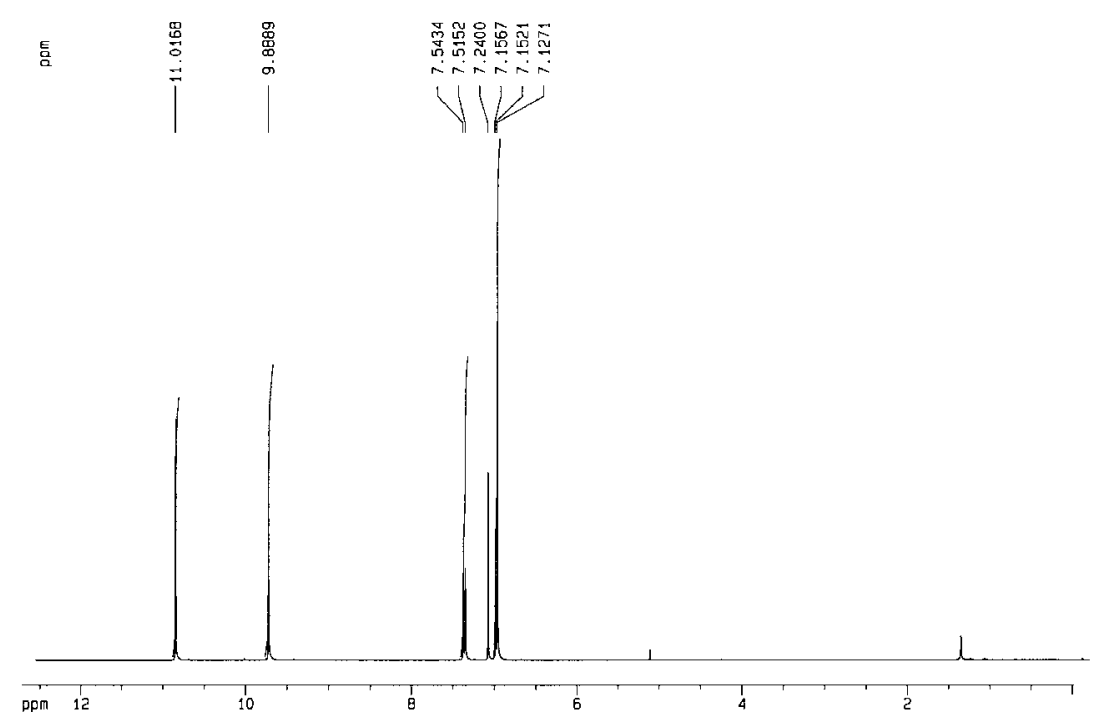

Figure S4. ${ }^{1} \mathrm{H}$ NMR spectrum of compound 3 .

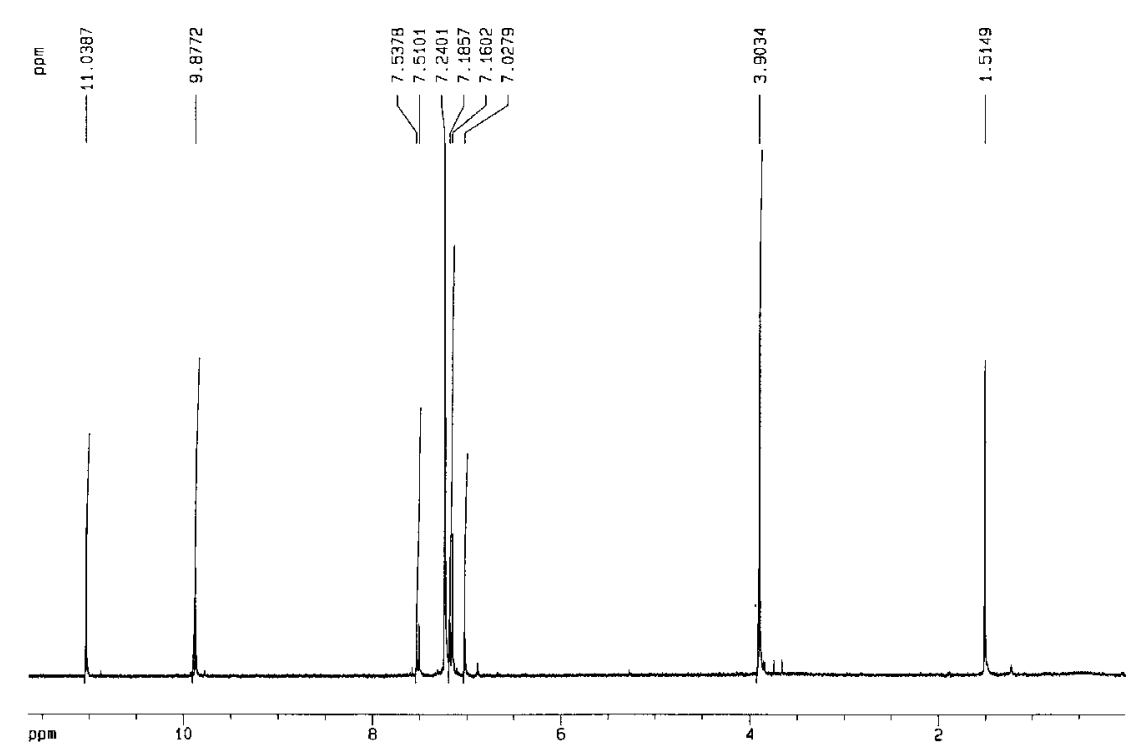

Figure S5. ${ }^{1} \mathrm{H}$ NMR spectrum of compound 4. 


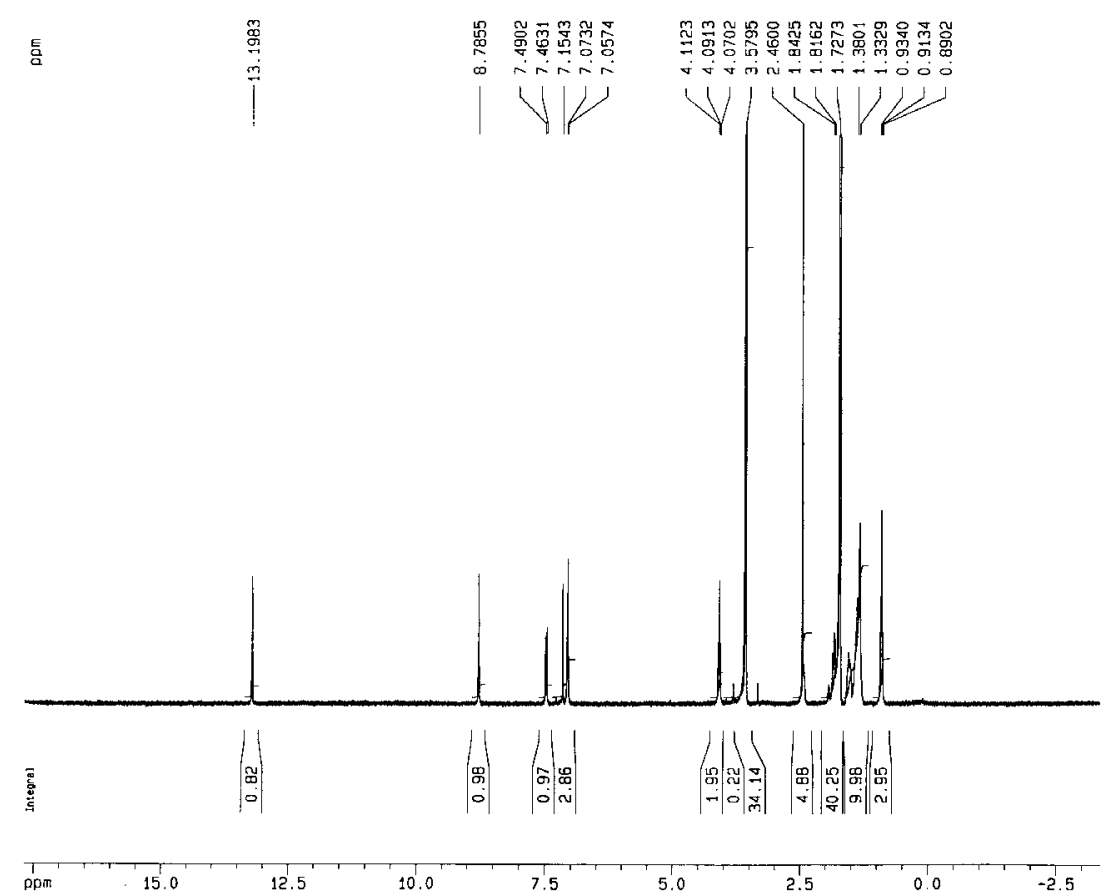

Figure S6. ${ }^{1} \mathrm{H}$ NMR spectrum of macrocycle 6 .

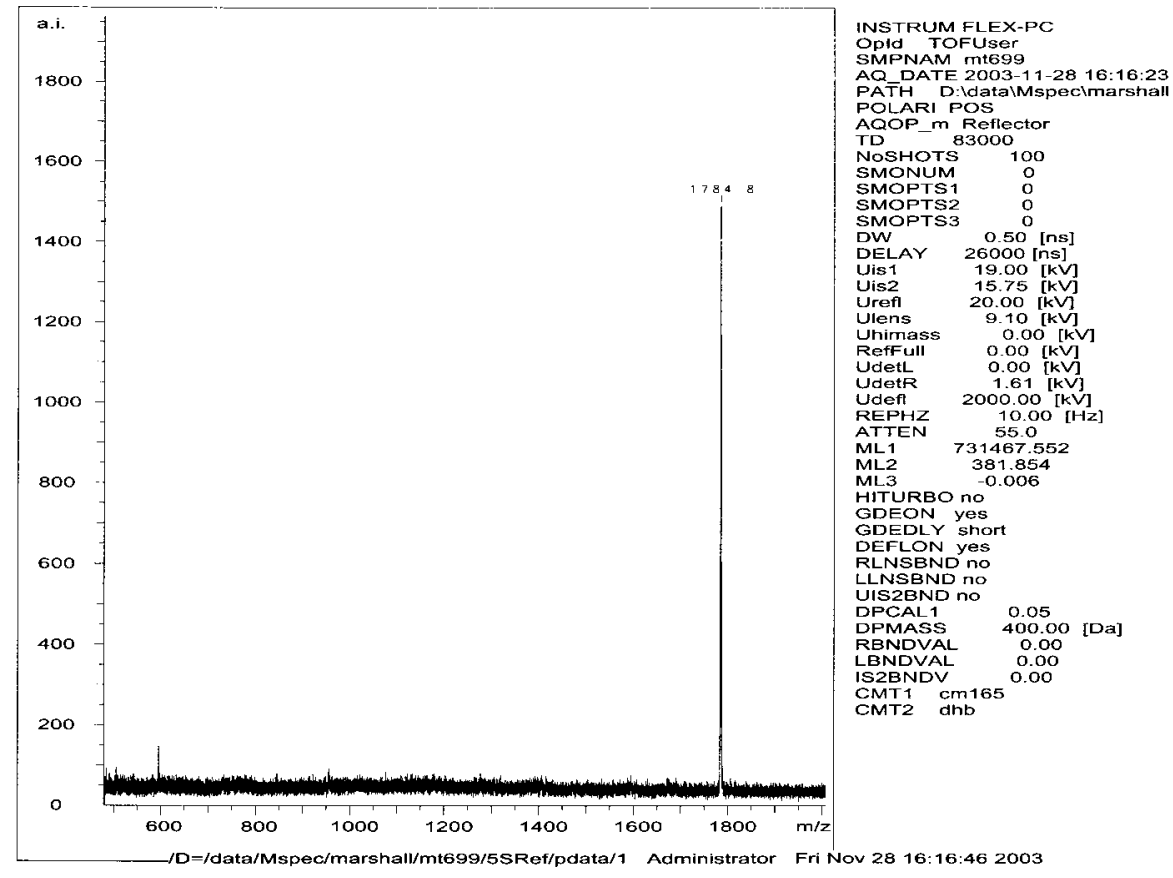

Figure S7. MALDI-TOF spectrum of macrocycle 6. 


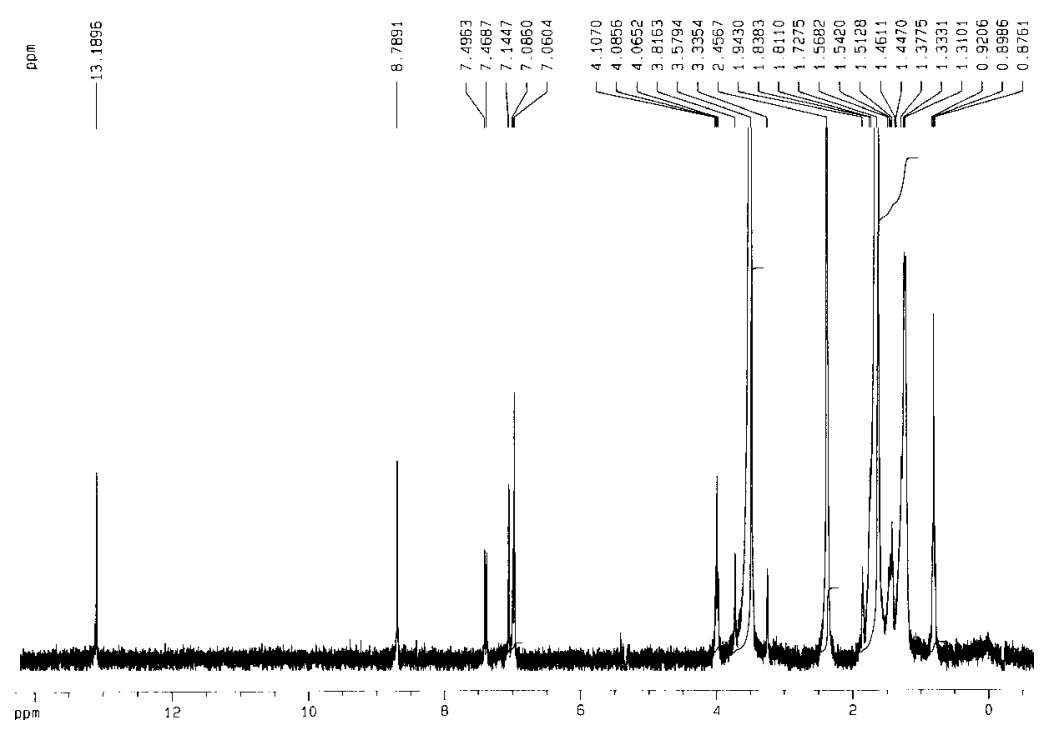

Figure S8. ${ }^{1} \mathrm{H}$ NMR spectrum of macrocycle 7.

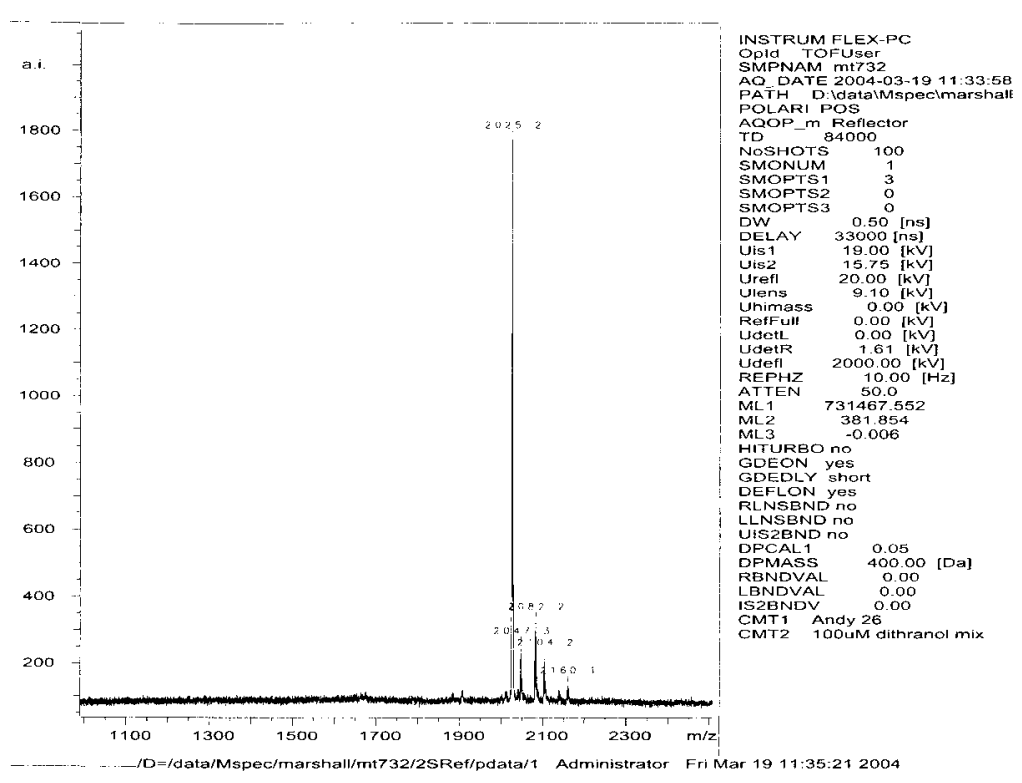

Figure S9. MALDI-TOF spectrum of macrocycle 7. 


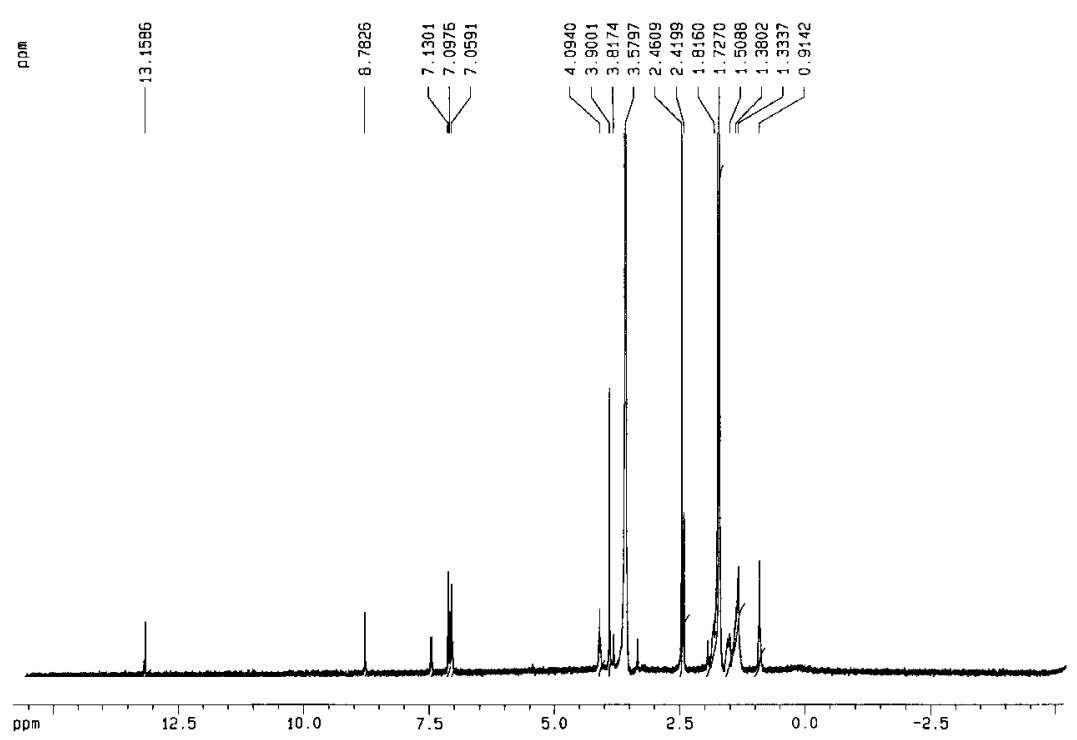

Figure S10. ${ }^{1} \mathrm{H}$ NMR spectrum of macrocycle 8 .

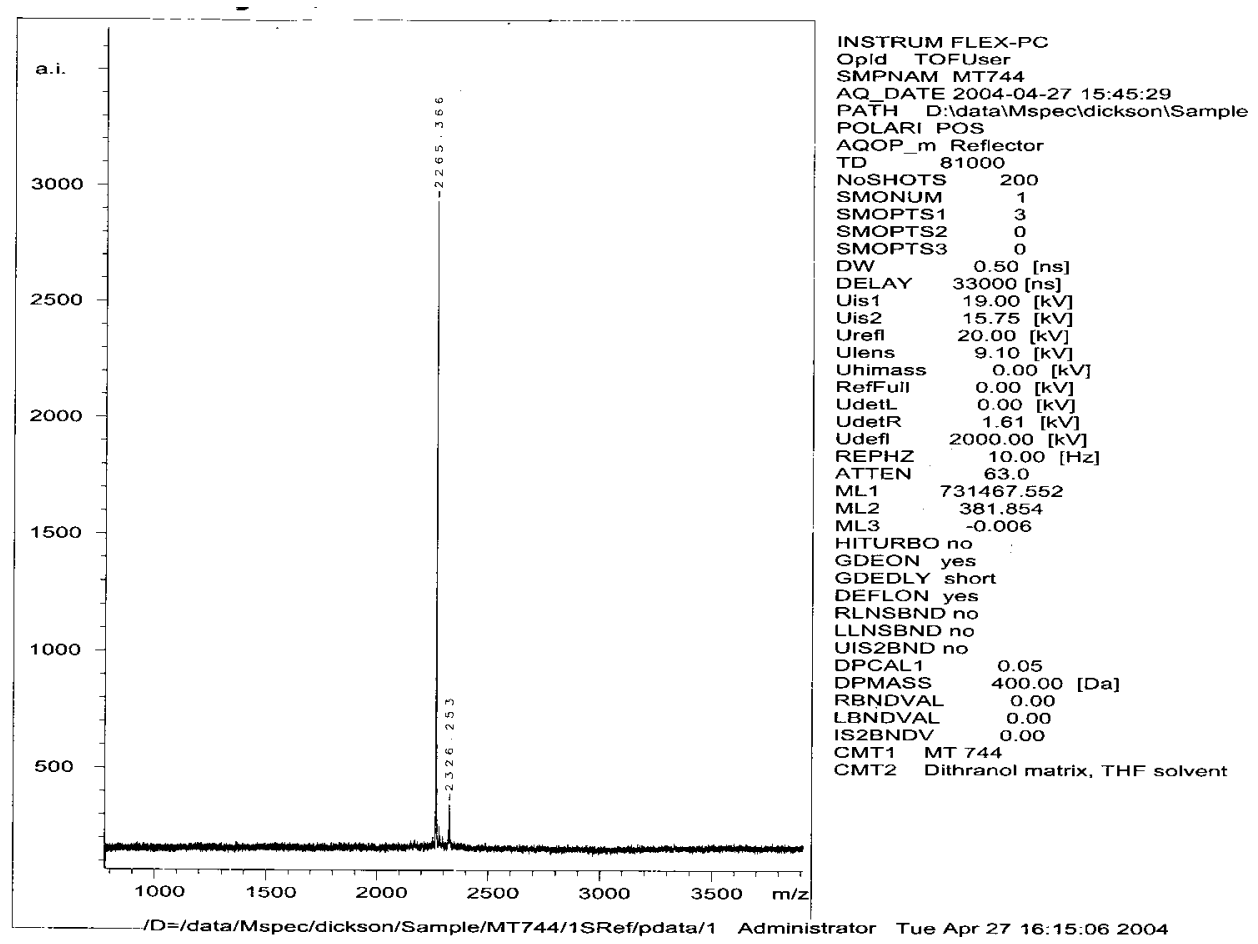

Figure S11. MALDI-TOF spectrum of macrocycle 8. 


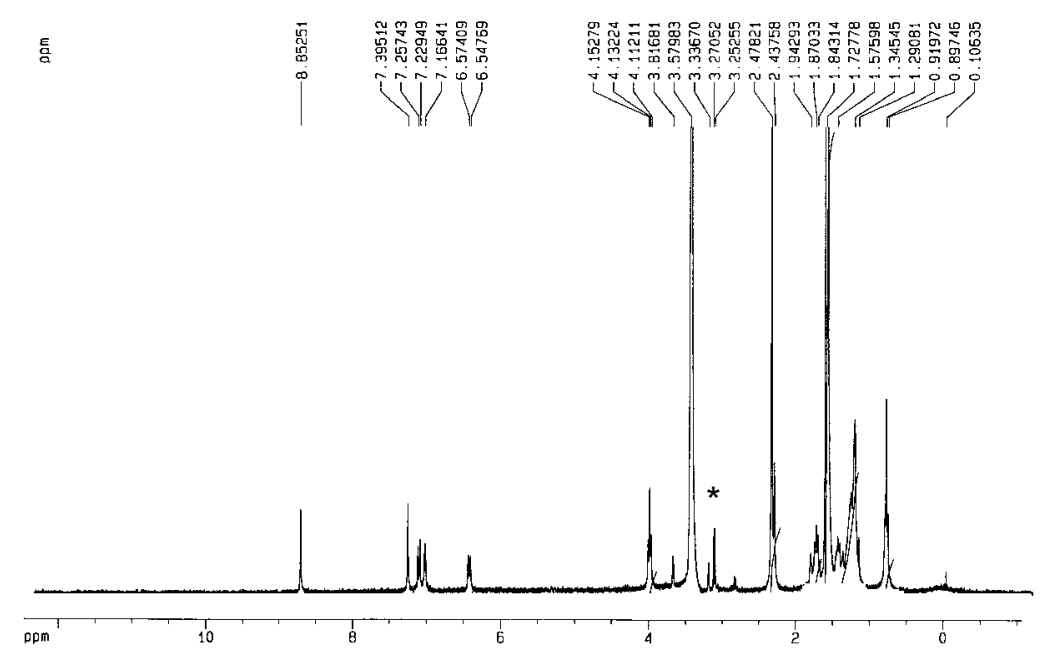

Figure S12. ${ }^{1} \mathrm{H}$ NMR spectrum of macrocycle $9(*=\mathrm{MeOH})$.

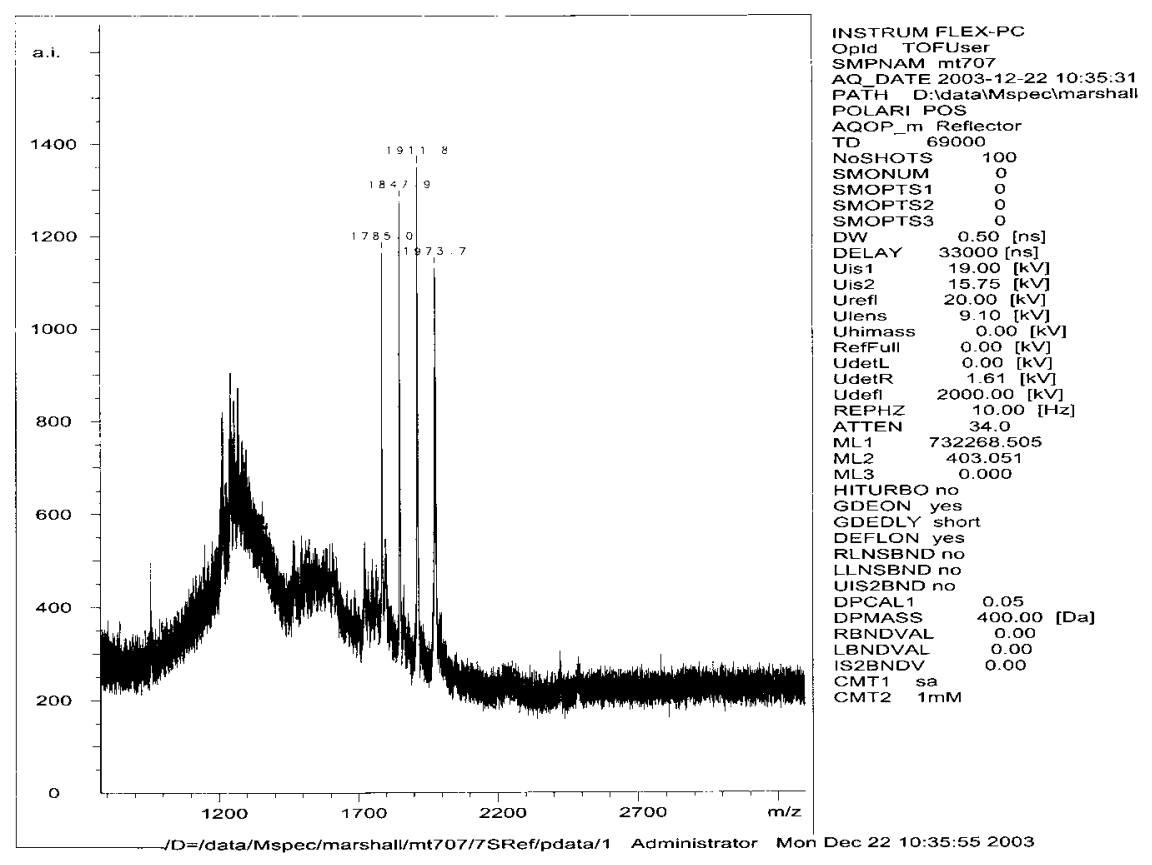

Figure S13. MALDI-TOF spectrum of macrocycle 9. 


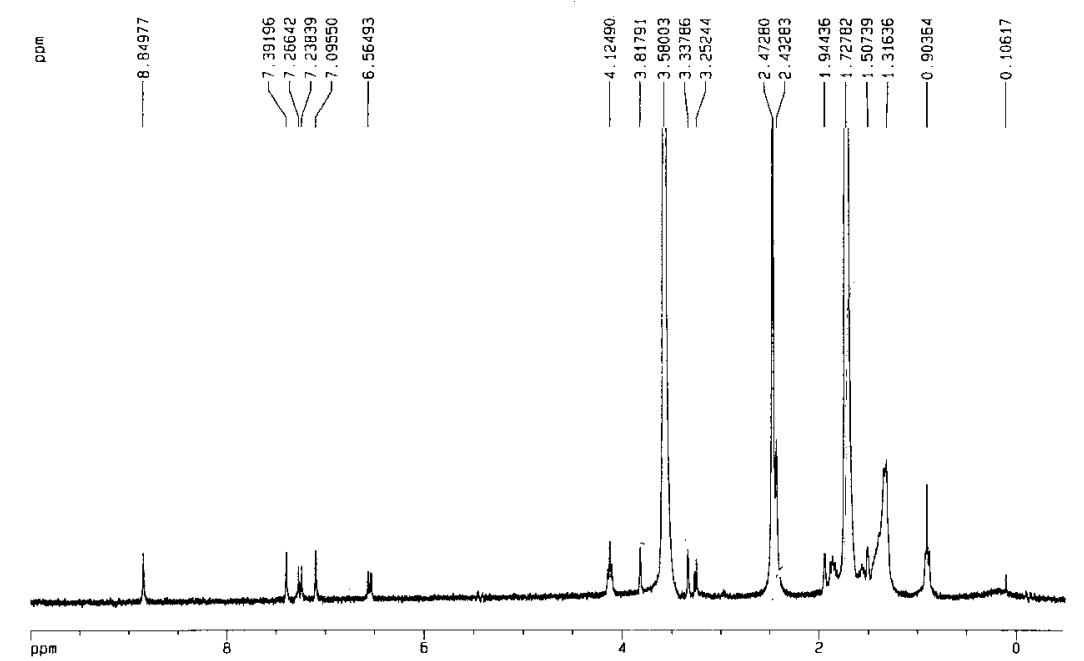

Figure S14. ${ }^{1}$ H NMR spectrum of macrocycle $\mathbf{1 0}$.

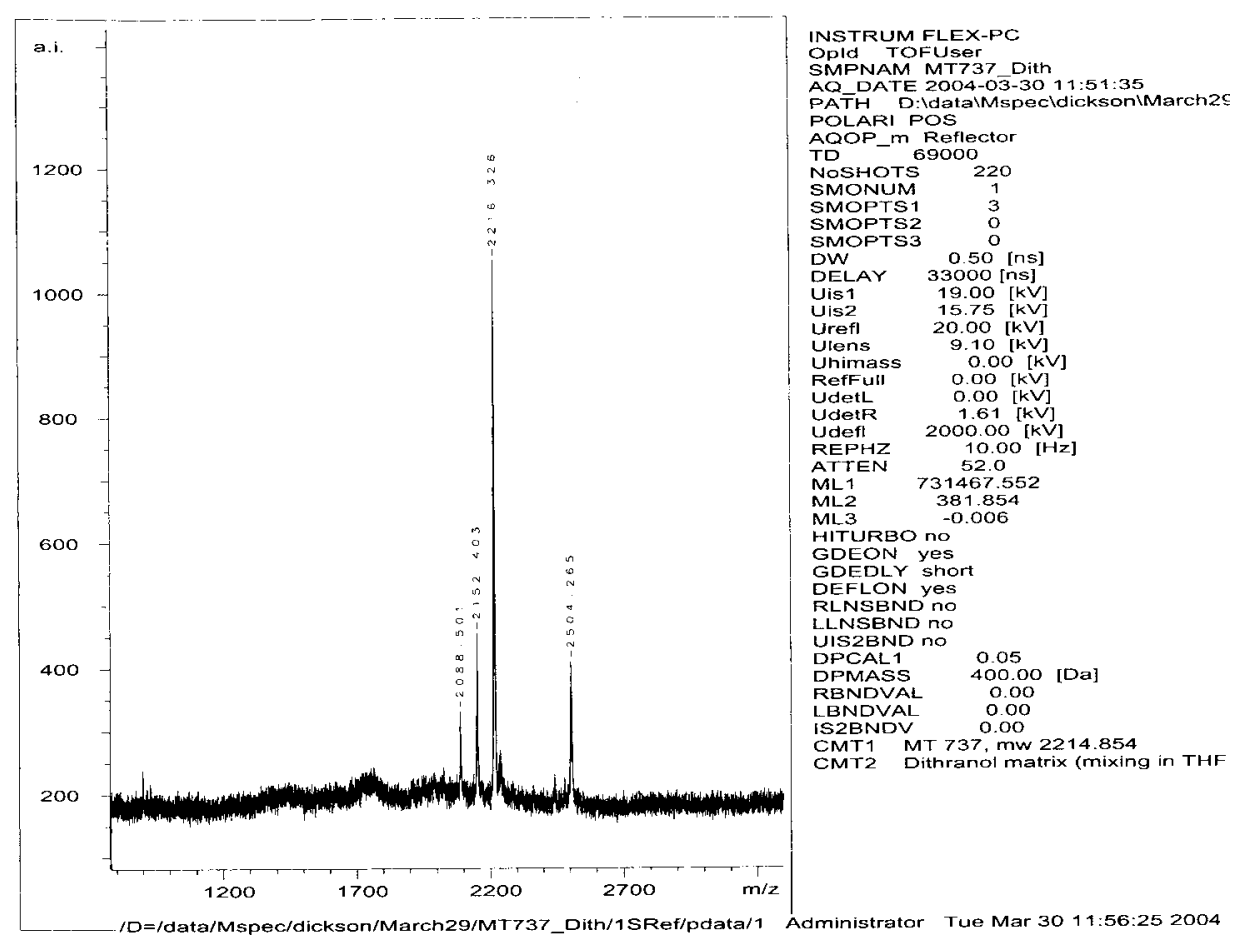

Figure S15. MALDI-TOF spectrum of macrocycle 10. 


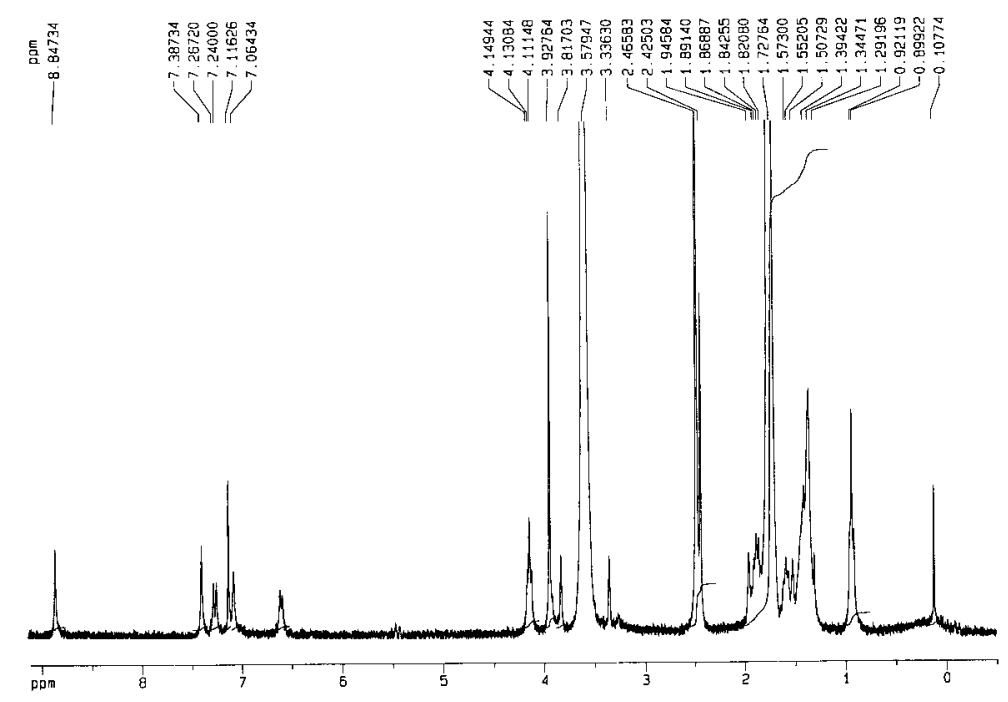

Figure S16. ${ }^{1}$ H NMR spectrum of macrocycle 11.

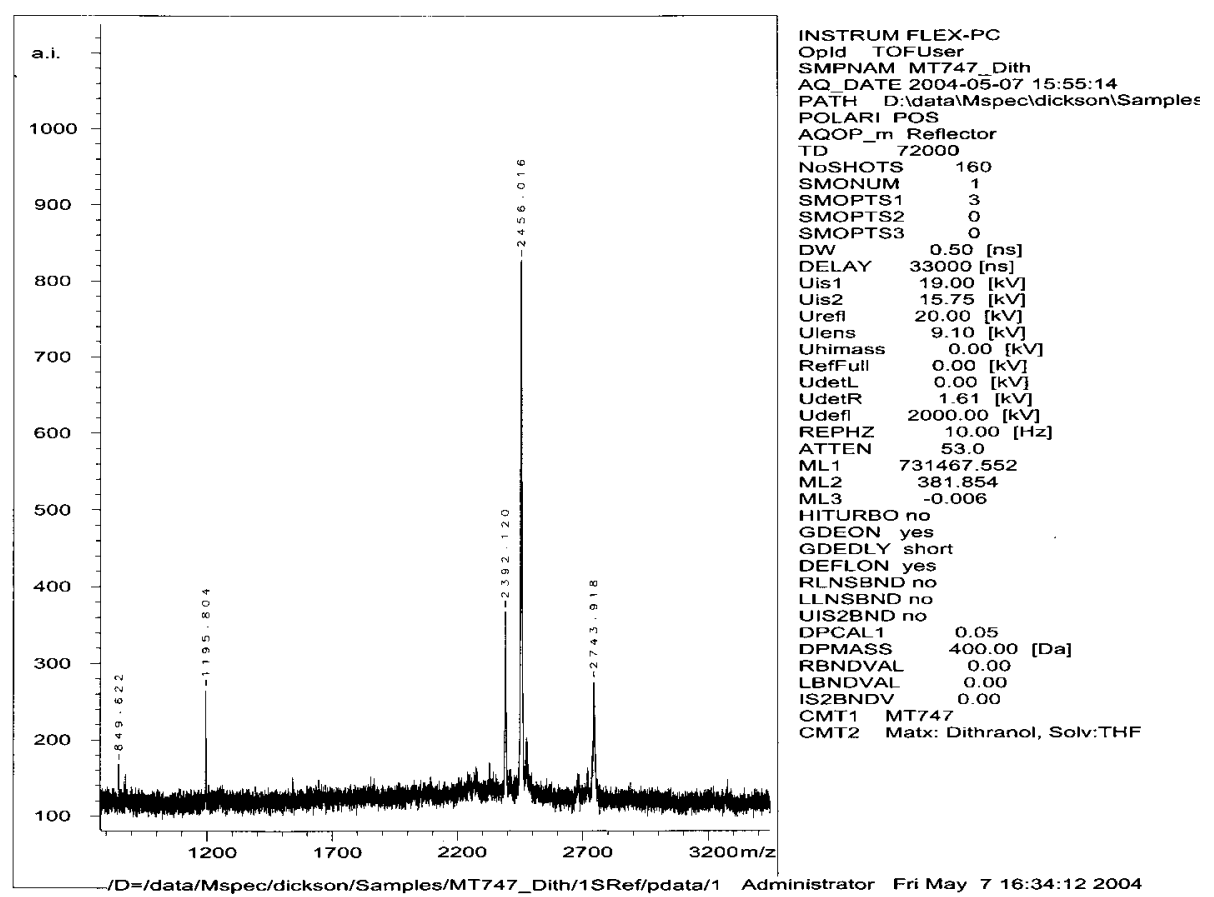

Figure S17. MALDI-TOF spectrum of macrocycle 11. 


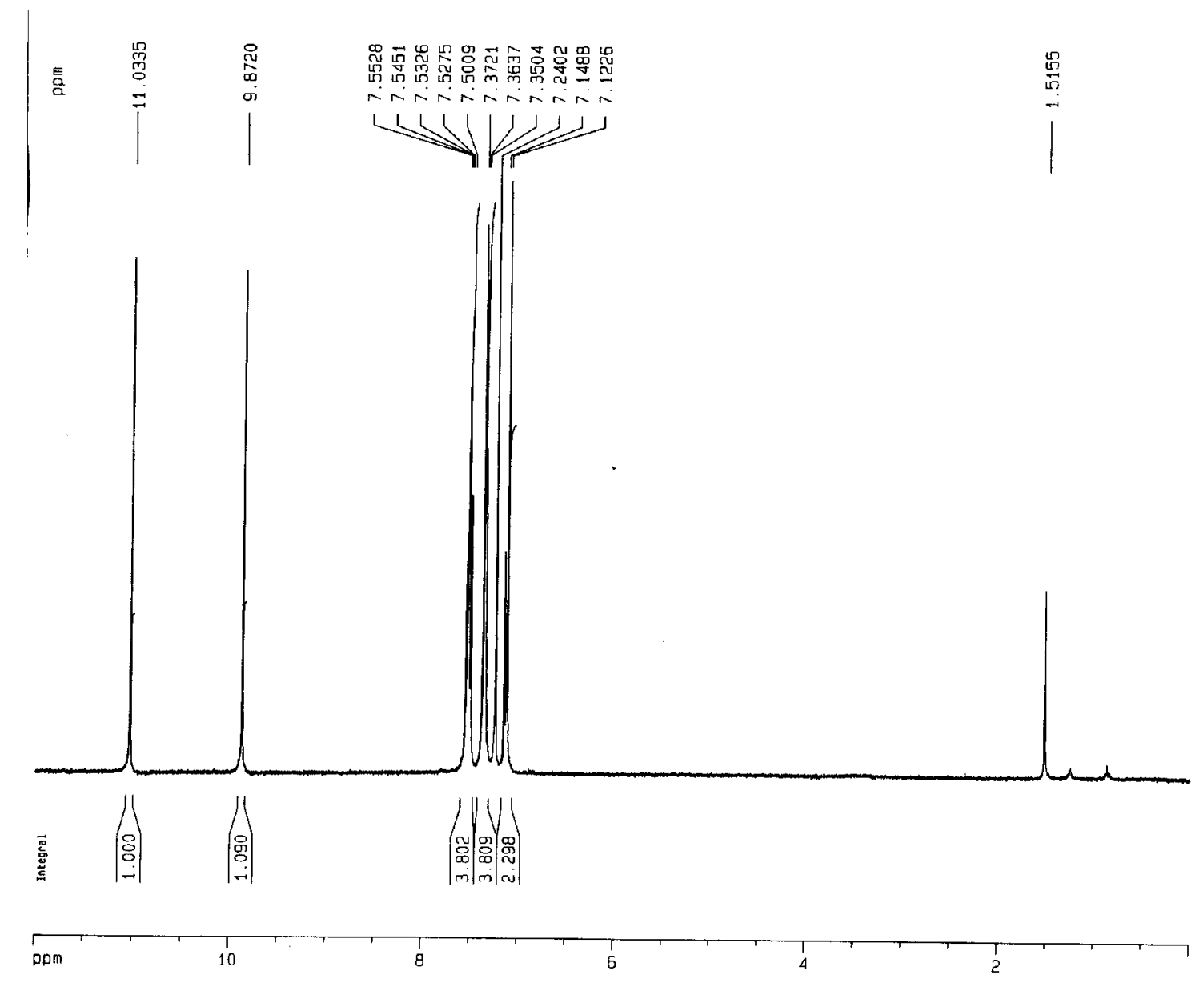

Figure S18. ${ }^{1} \mathrm{H}$ NMR spectrum of compound $12\left(\mathrm{CDCl}_{3}\right)$. 


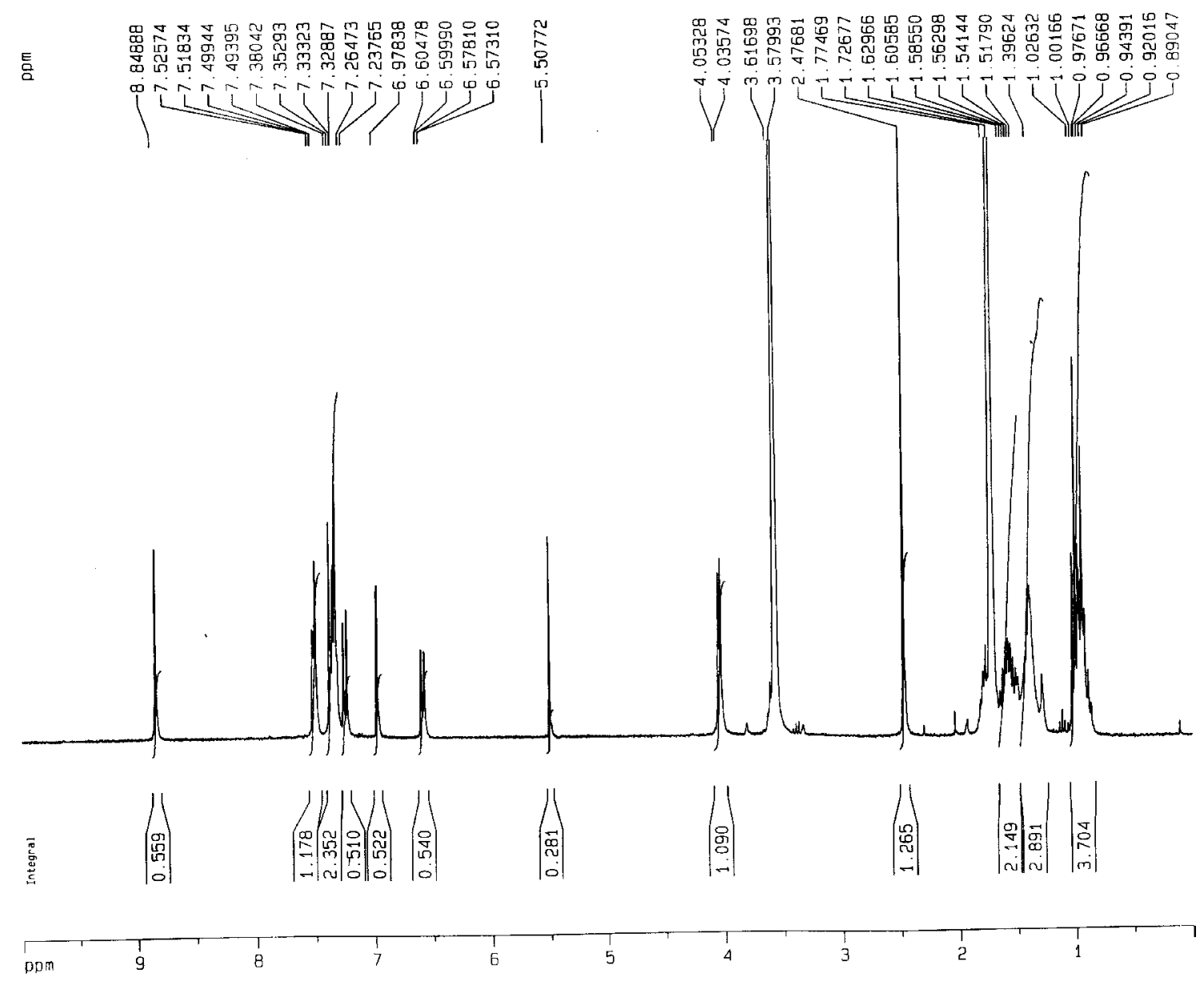

Figure S19. 1H NMR spectrum of model compound $13\left(\mathrm{THF}-\mathrm{d}_{8}\right)$. 


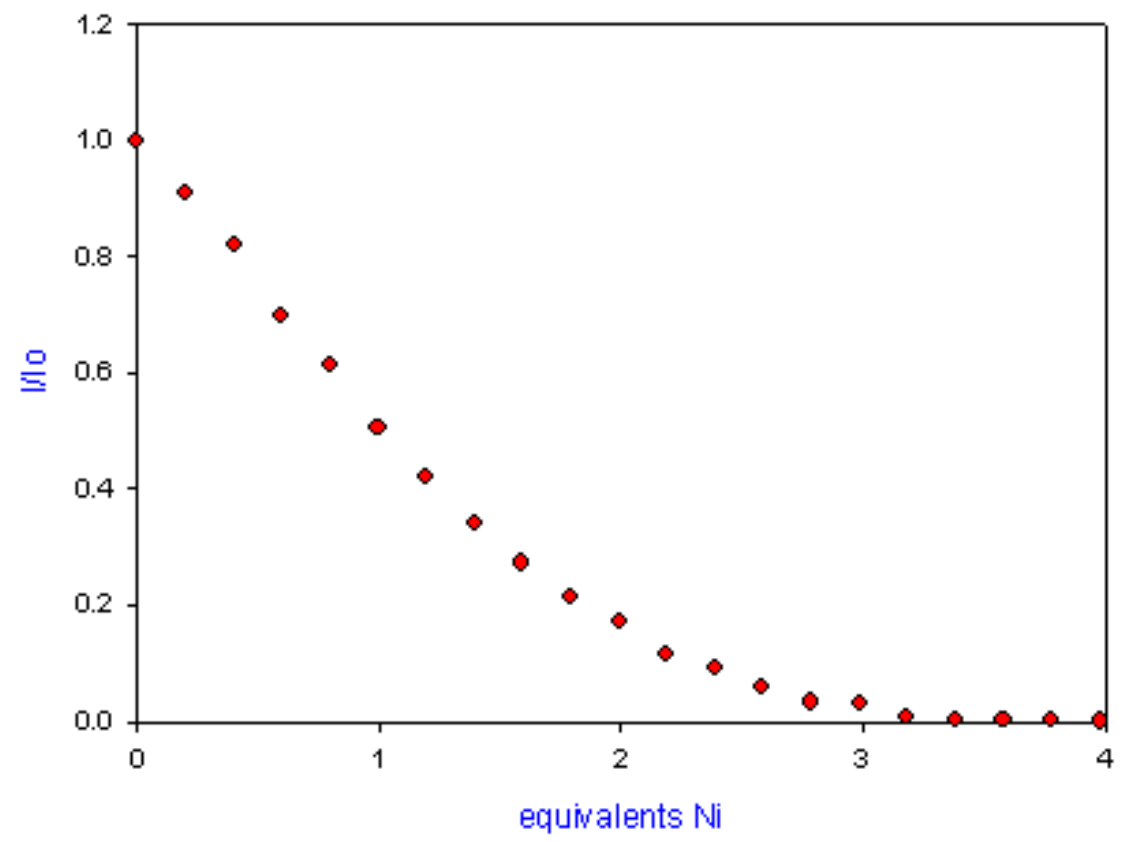

Figure S20. Graph of fluorescence intensity (normalized) vs. equiv. $\mathrm{Ni}(\mathrm{OAc})_{2}$ added to macrocycle 6 in THF. At 3 equiv, the fluorescence is fully quenched. The nonlinearity arises from the quenching or partial quenching of the fluorescence from macrocycles with 1 or 2 metals.

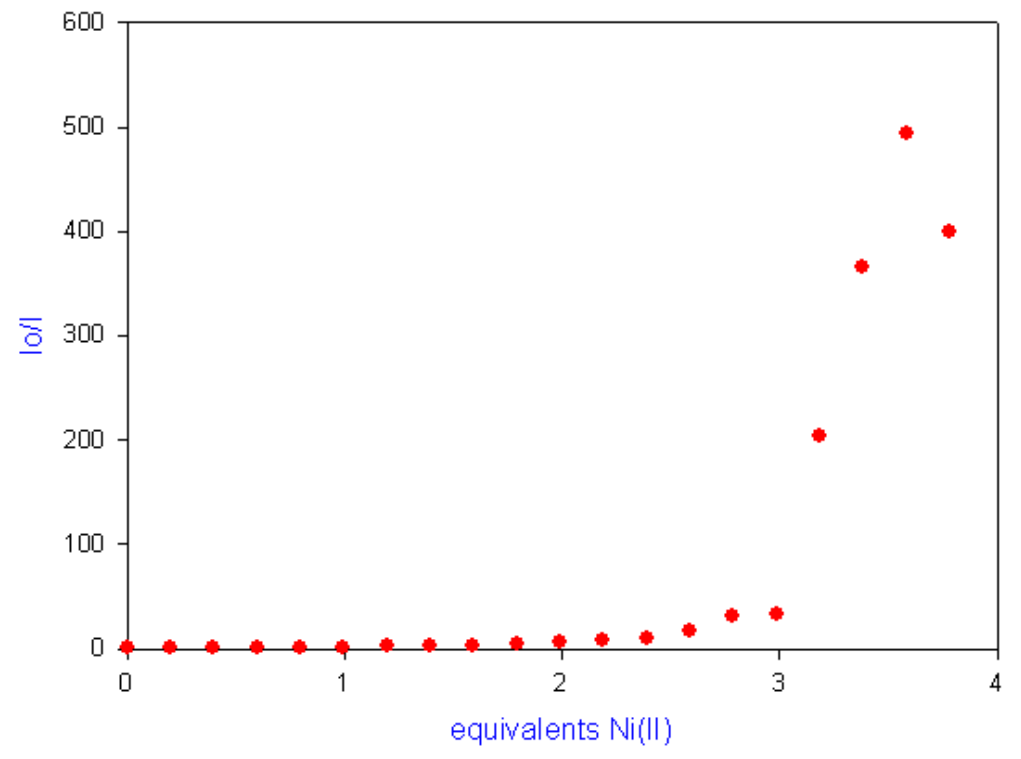

Figure S21. Stern-Volmer plot for the fluorescence quenching of macrocycle 6 . The plot of $\mathrm{I}_{\mathrm{o}} / \mathrm{I}$ should be linear in the case of dynamic quenching. The large positive curvature in the plot, as well as the near total quenching at 3 equiv. of $\mathrm{Ni}$ indicates that the quenching is static and complete at the stoichiometry of 3:1 $\mathrm{Ni}(\mathrm{II}): 6$. 